\title{
THEORY OF COSMOLOGICAL PERTURBATIONS AND APPLICATIONS TO SUPERSTRING COSMOLOGY
}

\author{
Robert H. Brandenberger \\ Physics Department, McGill University \\ 3600 rue Université \\ Montreal, QC, H3A 2T8, CANADA \\ and \\ Brown University Physics Department \\ Providence, RI 02912, USA \\ rhb@hep.physics.mcgill.ca
}

\begin{abstract}
The theory of cosmological perturbations is the main tool which connects theories of the early Universe (based on new fundamental physics such as string theory) with cosmological observations. In these lectures, I will provide an introduction to this theory, beginning with an overview of the Newtonian theory of fluctuations, moving on to the analysis of fluctuations in the realm of classical general relativity, and culminating with a discussion of the quantum theory of cosmological perturbations. I will illustrate the formalism with applications to inflationary cosmology. I will review the basics of inflationary cosmology and discuss why - through the evolution of fluctuations - inflation may provide a way of observationally testing Planck-scale physics.
\end{abstract}

\section{INTRODUCTION}

Recent years have provided a wealth of observational data about the cosmos. We have high resolution maps of the anisotropies in the temperature of the cosmic microwave background (CMB) [1], surveys of the large-scale structure (LSS) - the distribution of galaxies in threedimensional space - are increasing in size and in accuracy (see e.g. [2] and [3]), and new techniques which will allow us to measure the distri- 
bution of the dark matter are being pioneered. All of this data involves small deviations of the cosmos from homogeneity and isotropy. The cosmological observations reveal that the Universe has non-random fluctuations on all scales smaller than the present Hubble radius.

Parallel to this spectacular progress in observational cosmology, new cosmological scenarios have emerged within which it is possible to explain the origin of non-random inhomogeneities by means of causal physics. The scenario which has attracted most attention is inflationary cosmology $[4,5]$, according to which there was a period in the early Universe in which space was expanding at an accelerated rate. However, there are also alternative proposals $[7,8]$ in which our current stage of cosmological expansion is preceded by a phase of contraction. These scenarios have in common the fact that for scales of cosmological interest today, although their physical wavelength is larger than the Hubble length during most of the history of the universe, it is smaller than the Hubble radius at very early times, thus in principle allowing for a causal origin of the cosmological fluctuations.

In order to connect theories of fundamental physics providing an origin of perturbations with the data on the late time universe, one must be able to evolve cosmological fluctuations from earliest times to today. Since on large scales (scales larger than about $10 \mathrm{Mpc}-1 \mathrm{Mpc}$ being roughly three million light years) the relative density fluctuations are smaller than one today, and since these relative fluctuations grow in time as a consequence of gravitational instability, they were smaller than one throughout their history - at least in a universe which is always expanding. Thus, it is reasonable to expect that a linearized analysis of the fluctuations will give reliable results.

In most current models of the very early universe it is assumed that the perturbations originate as quantum vacuum fluctuations. Thus, quantum mechanics is important. On the other hand, since for most of the history of the universe the wavelengths corresponding to scales of cosmological interest today were larger than the Hubble radius, it is crucial to consider the general relativistic theory of fluctuations. Hence, a quantum theory of general relativistic fluctuations is required. At the level of the linearized theory of cosmological perturbations, a unified quantum theory of the formation and evolution of fluctuations exists - and this will be the main topic of these lectures. Note that all of the conceptual problems of merging quantum mechanics and general relativity have been thrown out by hand by restricting attention to the linearized analysis. The question of "linearization stability" of the system, namely whether the solutions of the linearized equations of motion in fact correspond to the linearization of solutions of the full equations, is a deep 
one and will not be addressed here (see e.g. [6] for discussions of this issue).

Inflationary cosmology is at the present time the most successful framework of connecting physics of the very early universe with the present structure (although alternatives such as the Pre-Big-Bang [7] and Ekpyrotic [8] scenarios have been proposed and may turn out to be successful as well). I will thus begin these lectures with a review of inflationary cosmology, and of scalar field-driven models for inflation. Next, I will provide a detailed discussion of the theory of cosmological perturbations, beginning with the Newtonian theory, moving on to the classical general relativistic analysis, and ending with the quantum theory of cosmological perturbations ${ }^{1}$ In the final sections of these notes, I will return to inflationary cosmology, and focus on some important conceptual problems which are not addressed in current realizations of inflation in which the accelerated expansion of space is driven by a scalar matter field. Addressing these conceptual problems is a challenge and great opportunity for string theory. Since cosmological inflation leads to a quasi-exponential increase in the wavelength of inhomogeneities, it provides a microscope with which string-scale physics can in principle be probed in current observations. I will conclude these lectures with a discussion of this "window of opportunity" for string theory which inflation provides [9].

\section{OVERVIEW OF INFLATIONARY COSMOLOGY}

To establish our notation and framework, we will be taking the background space-time to be homogeneous and isotropic, with a metric given by

$$
d s^{2}=d t^{2}-a(t)^{2} d \mathbf{x}^{2},
$$

where $t$ is physical time, $d \mathbf{x}^{2}$ is the Euclidean metric of the spatial hypersurfaces (here taken for simplicity to be spatially flat), and $a(t)$ is the scale factor. The scale factor determines the Hubble expansion rate via

$$
H(t)=\frac{\dot{a}}{a}(t)
$$

The coordinates $\mathbf{x}$ used above are comoving coordinates, coordinates painted onto the expanding spatial hypersurfaces.

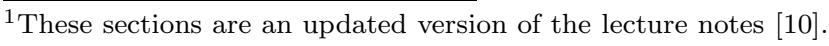


In standard big bang cosmology, the universe is decelerating, i.e. $\ddot{a}<$ 0 . As a consequence, the Hubble radius

$$
l_{H}(t)=H^{-1}(t)
$$

is increasing in comoving coordinates. As will be explained later mathematically, the Hubble radius is the maximal distance that microphysics can act coherently over a Hubble expansion time - in particular it is the maximal distance on which any causal process could create fluctuations. If the universe were decelerating forever, then scales of cosmological interest today would have had a wavelength larger than the Hubble radius at all early times. This gives rise to the fluctuation problem for Standard Big Bang (SBB) cosmology, namely the problem that there cannot be any causal process which at early time creates perturbations on scales which are being probed in current LSS and CMB observations ${ }^{2}$.

The idea of inflationary cosmology is to assume that there was a period in the very early Universe during which the scale factor was accelerating, i.e. $\ddot{a}>0$. This implies that the Hubble radius was shrinking in comoving coordinates, or, equivalently, that fixed comoving scales were "exiting" the Hubble radius. In the simplest models of inflation, the scale factor increases nearly exponentially. As illustrated in Figure (1.1), the basic geometry of inflationary cosmology provides a solution of the fluctuation problem. As long as the phase of inflation is sufficiently long, all length scales within our present Hubble radius today originate at the beginning of inflation with a wavelength smaller than the Hubble radius at that time. Thus, it is possible to create perturbations locally using physics obeying the laws of special relativity (in particular causality). As will be discussed later, it is quantum vacuum fluctuations of matter fields and their associated curvature perturbations which are responsible for the structure we observe today.

Postulating a phase of inflation in the very early universe also solves the horizon problem of the SBB, namely it explains why the causal horizon at the time $t_{r e c}$ when photons last scatter is larger than the radius of the past light cone at $t_{r e c}$, the part of the last scattering surface which is visible today in CMB experiments. Inflation also explains the near flatness of the universe: in a decelerating universe spatial flatness is an unstable fixed point of the dynamics, whereas in an accelerating universe it becomes an attractor. Another important aspect of the inflationary solution of the flatness problem is that inflation exponentially increases the volume of space. Without inflation, it is not possible that a Planck

${ }^{2}$ The reader is encouraged to find the hole in this argument, and is referred to [11, 12, 13] for the answer. 


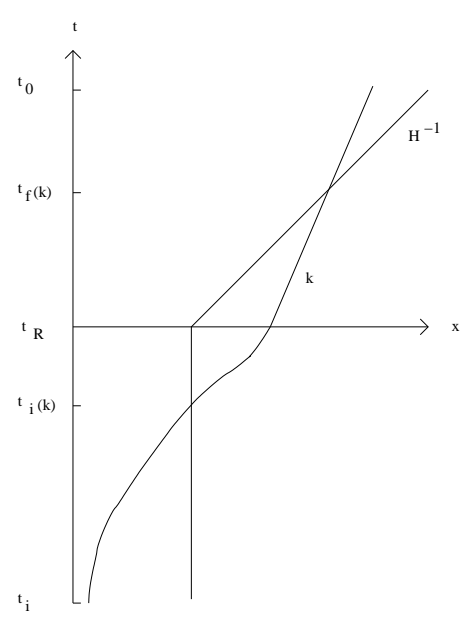

Figure 1.1 Space-time diagram (sketch) showing the evolution of scales in inflationary cosmology. The vertical axis is time, and the period of inflation lasts between $t_{i}$ and $t_{R}$, and is followed by the radiation-dominated phase of standard big bang cosmology. During exponential inflation, the Hubble radius $H^{-1}$ is constant in physical spatial coordinates (the horizontal axis), whereas it increases linearly in time after $t_{R}$. The physical length corresponding to a fixed comoving length scale labelled by its wavenumber $k$ increases exponentially during inflation but increases less fast than the Hubble radius (namely as $t^{1 / 2}$ ), after inflation.

scale universe at the Planck time evolves into a sufficiently large universe today.

Let us now consider how it is possible to obtain a phase of cosmological inflation. We will assume that space-time is to be described using the equations of General Relativity ${ }^{3}$. In this case, the dynamics of the scale factor $a(t)$ is determined by the Friedmann-Robertson-Walker (FRW) equations

$$
\left(\frac{\dot{a}}{a}\right)^{2}=8 \pi G \rho
$$

and

$$
\frac{\ddot{a}}{a}=-4 \pi G(\rho+3 p)
$$

where for simplicity we have omitted the contributions of spatial curvature (since spatial curvature is diluted during inflation) and of the cosmological constant (since any small cosmological constant which might be present today has no effect in the early Universe since the associated energy density does not increase when going into the past). In the above,

${ }^{3}$ Note, however, that the first model of exponential expansion of space [14] made use of a higher derivative gravitational action. 
$\rho$ and $p$ denote the energy density and pressure, respectively. From (1.5) it is clear that in order to obtain an accelerating universe, matter with sufficiently negative pressure

$$
p<-\frac{1}{3} \rho
$$

is required. Exponential inflation is obtained for $p=-\rho$.

Conventional perfect fluids have positive semi-definite pressure and thus cannot yield inflation. In addition, we know that a description of matter in terms of classical perfect fluids must break down at early times. An improved description of matter will be given in terms of quantum fields. Scalar matter fields are special in that they allow at the level of a renormalizable action the presence of a potential energy term. The energy density and pressure of a scalar field $\varphi$ with canonically normalized action 4

$$
\mathcal{L}=\sqrt{-g}\left[\frac{1}{2} \partial_{\mu} \varphi \partial^{\mu} \varphi-V(\varphi)\right]
$$

(where Greek indices are space-time indices, $V(\varphi)$ is the potential energy density, and $g$ is the determinant of the metric) are given by

$$
\begin{aligned}
\rho & =\frac{1}{2}(\dot{\varphi})^{2}+\frac{1}{2} a^{-2}(\nabla \varphi)^{2}+V(\varphi) \\
p & =\frac{1}{2}(\dot{\varphi})^{2}-\frac{1}{6} a^{-2}(\nabla \varphi)^{2}-V(\varphi) .
\end{aligned}
$$

Thus, it is possible to obtain an almost exponentially expanding universe provided the scalar field configuration ${ }^{5}$ satisfies

$$
\begin{aligned}
\frac{1}{2}\left(\nabla_{p} \varphi\right)^{2} & \ll V(\varphi), \\
\frac{1}{2}(\dot{\varphi})^{2} & \ll V(\varphi) .
\end{aligned}
$$

In the above, $\nabla_{p} \equiv a^{-1} \nabla$ is the gradient with respect to physical as opposed to comoving coordinates. Since spatial gradients redshift as the universe expands, the first condition will (for single scalar field models) always be satisfied if it is satisfied at the initial time ${ }^{6}$. It is the second condition which is harder to satisfy. In particular, this condition is in general not preserved in time even it is initially satisfied.

\footnotetext{
${ }^{4}$ See [15] for a discussion of fields with non-canonical kinetic terms.

${ }^{5}$ The scalar field yielding inflation is called the inflaton.

${ }^{6}$ In fact, careful studies [16] show that since the gradients decrease even in a non-inflationary backgrounds, they can become subdominant even if they are not initially subdominant.
} 
It is sufficient to obtain a period of cosmological inflation that the slow-roll conditions for $\varphi$ are satisfied. Recall that the equation of motion for a homogeneous scalar field in a cosmological space-time is (as follows from (1.7)) is

$$
\ddot{\varphi}+3 H \dot{\varphi}=-V^{\prime}(\varphi),
$$

where a prime indicates the derivative with respect to $\varphi$. In order that the scalar field roll slowly, it is necessary that

$$
\ddot{\varphi} \ll 3 H \dot{\varphi}
$$

such that the first term in the scalar field equation of motion (1.11) is negligible. In this case, the condition (1.10) becomes

$$
\left(\frac{V^{\prime}}{V}\right)^{2} \ll 48 \pi G
$$

and (1.12) becomes

$$
\frac{V^{\prime \prime}}{V} \ll 24 \pi G
$$

In the initial model of inflation using scalar fields ("old inflation" [4]), it was assumed that $\varphi$ was initially in a false vacuum with large potential energy. Hence, the conditions for inflation are trivially satisfied. To end inflation, a quantum tunneling event from the false vacuum to the true vacuum [17] was invoked (see e.g. [18] for a pedagogical review). This model, however, has a graceful exit problem since the tunneling leads to an initially microscopical bubble of the true vacuum which cannot grow to encompass our presently observed universe - the flatness problem of SBB cosmology in a new form. Hence, attention shifted to models in which the scalar field $\varphi$ is slowly rolling during inflation.

There are many models of scalar field-driven inflation. Many of them can be divided into three groups [19]: small-field inflation, large-field inflation and hybrid inflation. Small-field inflationary models are based on ideas from spontaneous symmetry breaking in particle physics. We take the scalar field to have a potential of the form

$$
V(\varphi)=\frac{1}{4} \lambda\left(\varphi^{2}-\sigma^{2}\right)^{2},
$$

where $\sigma$ can be interpreted as a symmetry breaking scale, and $\lambda$ is a dimensionless coupling constant. The hope of initial small-field models ("new inflation" [20]) was that the scalar field would begin rolling close to its symmetric point $\varphi=0$, where thermal equilibrium initial conditions would localize it in the early universe. At sufficiently high temperatures, 
$\varphi=0$ is a stable ground state of the one-loop finite temperature effective potential $V_{T}(\varphi)$ (see e.g. [18] for a review). Once the temperature drops to a value smaller than the critical temperature $T_{c}, \varphi=0$ turns into an unstable local maximum of $V_{T}(\varphi)$, and $\varphi$ is free to roll towards a ground state of the zero temperature potential (1.15). The direction of the initial rolling is triggered by quantum fluctuations. The reader can easily check that for the potential (1.15) the slow-roll conditions cannot be satisfied if $\sigma \ll m_{p l}$, where $m_{p l}$ is the Planck mass which is related to $G$. If the potential is modified to a Coleman-Weinberg [21] form

$$
V(\varphi)=\frac{\lambda}{4} \varphi^{4}+\frac{\lambda^{2}}{44 \pi^{2}} \varphi^{4}\left[\ln \left(\frac{\varphi^{2}}{M^{2}}\right)-\frac{25}{6}\right]
$$

(where $M$ denotes some renormalization scale) then the slow-roll conditions can be satisfied. However, this corresponds to a severe fine-tuning of the shape of the potential. A further problem for most small-field models of inflation (see e.g. [22] for a review) is that the slow-roll trajectory is not an attractor in phase space. In order to end up close to the slow-roll trajectory, the initial field velocity must be constrained to be very small. This initial condition problem of small-field models of inflation effects a number of recently proposed brane inflation scenarios, see e.g. [23] for a discussion.

There is another reason for abandoning small-field inflation models: in order to obtain a sufficiently small amplitude of density fluctuations, the interaction coefficients of $\varphi$ must be very small (this problem is discussed at length towards the end of these lectures). In particular, this makes it inconsistent to assume that $\varphi$ started out in thermal equilibrium. In the absence of thermal equilibrium, the phase space of initial conditions is much larger for large values of $\varphi$.

This brings us to the discussion of large-field inflation models, initially proposed in [24] under the name "chaotic inflation". The simplest example is provided by a massive scalar field with potential

$$
V(\varphi)=\frac{1}{2} m^{2} \varphi^{2}
$$

where $m$ is the mass. It is assumed that the scalar field rolls towards the origin from large values of $|\varphi|$. It is a simple exercise for the reader to verify that the slow-roll conditions (1.13) and (1.14) are satisfied provided

$$
|\varphi|>\frac{1}{\sqrt{12 \pi}} m_{p l}
$$

Values of $|\varphi|$ comparable or greater than $m_{p l}$ are also required in other realizations of large-field inflation. Hence, one may worry whether such 
a toy model can consistently be embedded in a realistic particle physics model, e.g. supergravity. In many such models $V(\varphi)$ receives supergravityinduced correction terms which destroy the flatness of the potential for $|\varphi|>m_{p l}$. However, as recently discussed in [25], if the flatness of the potential is protected by some symmetry, then it can survive inclusion of the correction terms. As will be discussed later, a value of $m \sim 10^{13} \mathrm{GeV}$ is required in order to obtain the observed amplitude of density fluctuations. Hence, the configuration space of field values with $|\varphi|>m_{p l}$ but $V(\varphi)<m_{p l}^{4}$ is huge. It can also be verified that the slow-roll trajectory is a local attractor in field initial condition space [16], even including metric fluctuations at the perturbative level [26].

With two scalar fields it is possible to construct a class of models which combine some of the nice features of large-field inflation (large phase space of initial conditions yielding inflation) and of small-field inflation (better contact with conventional particle physics). These are models of hybrid inflation [27]. To give a prototypical example, consider two scalar fields $\varphi$ and $\chi$ with a potential

$$
V(\varphi, \chi)=\frac{1}{4} \lambda_{\chi}\left(\chi^{2}-\sigma^{2}\right)^{2}+\frac{1}{2} m^{2} \varphi^{2}-\frac{1}{2} g^{2} \varphi^{2} \chi^{2}
$$

In the absence of thermal equilibrium, it is natural to assume that $|\varphi|$ begins at large values, values for which the effective mass of $\chi$ is positive and hence $\chi$ begins at $\chi=0$. The parameters in the potential (1.19) are now chosen such that $\varphi$ is slowly rolling for values of $|\varphi|$ somewhat smaller than $m_{p l}$, but that the potential energy for these field values is dominated by the first term on the right-hand side of (1.19). The reader can easily verify that for this model it is no longer required to have values of $|\varphi|$ greater than $m_{p l}$ in order to obtain slow-rolling ${ }^{7}$ The field $\varphi$ is slowly rolling whereas the potential energy is determined by the contribution from $\chi$. Once $|\varphi|$ drops to the value

$$
\left|\varphi_{c}\right|=\frac{\sqrt{\lambda_{\chi}}}{g} \sigma
$$

the configuration $\chi=0$ becomes unstable and decays to its ground state $|\chi|=\sigma$, yielding a graceful exit from inflation. Since in this example the ground state of $\chi$ is not unique, there is the possibility of the formation of topological defects at the end of inflation (see $[11,12,13]$ for reviews of topological defects in cosmology, and the lectures by Polchinski at this

${ }^{7}$ Note that the slow-roll conditions (1.13) and (1.14) were derived assuming that $H$ is given by the contribution of $\varphi$ to $V$ which is not the case here. 
school [28] for a discussion of how this scenario arises in brane inflation models).

After the slow-roll conditions break down, the period of inflation ends, and the inflaton begins to oscillate around its ground state. Due to couplings of $\varphi$ to other matter fields, the energy of the universe, which at the end of the period of inflation is stored completely in $\varphi$, gets transferred to the matter fields of the particle physics Standard Model. Initially, the energy transfer was described perturbatively [29, 30]. Later, it was realized [31, 32, 33, 34] that through a parametric resonance instability, particles are very rapidly produced, leading to a fast energy transfer ("preheating"). The quanta later thermalize, and thereafter the universe evolves as described by SBB cosmology.

After this review of inflationary cosmology (see e.g. [35] for a more complete recent review), we turn to the discussion of the main success of inflationary cosmology, namely the fact that it provides a causal mechanism for generating small inhomogeneities. The reader is referred to [36] for a comprehensive analysis of this theory of cosmological perturbations.

\section{NEWTONIAN THEORY OF COSMOLOGICAL PERTURBATIONS \\ 3.1 INTRODUCTION}

The growth of density fluctuations is a consequence of the purely attractive nature of the gravitational force. Imagine (first in a nonexpanding background) a density excess $\delta \rho$ localized about some point $\mathbf{x}$ in space. This fluctuation produces an attractive force which pulls the surrounding matter towards $\mathbf{x}$. The magnitude of this force is proportional to $\delta \rho$. Hence, by Newton's second law

$$
\ddot{\delta} \rho \sim G \delta \rho
$$

where $G$ is Newton's gravitational constant. Hence, there is an exponential instability of flat space-time to the development of fluctuations.

Obviously, in General Relativity it is inconsistent to consider density fluctuations in a non-expanding background. If we consider density fluctuations in an expanding background, then the expansion of space leads to a friction term in (1.21). Hence, instead of an exponential instability to the development of fluctuations, the growth rate of fluctuations in an expanding Universe will be as a power of time. It is crucial to determine what this power is and how it depends both on the background cosmological expansion rate and on the length scale of the fluctuations. 
Note that in the following two subsections $\mathbf{x}$ will denote the physical coordinates, and $\mathbf{q}$ the comoving ones. The materials covered in this section are discussed in several excellent textbooks on cosmology, e.g. in $[37,38,39,40]$.

\subsection{PERTURBATIONS ABOUT MINKOWSKI SPACE-TIME}

To develop some physical intuition, we first consider the evolution of hydrodynamical matter fluctuations in a fixed non-expanding background.

In this context, matter is described by a perfect fluid, and gravity by the Newtonian gravitational potential $\varphi$. The fluid variables are the energy density $\rho$, the pressure $p$, the fluid velocity $\mathbf{v}$, and the entropy density $S$. The basic hydrodynamical equations are

$$
\begin{aligned}
\dot{\rho}+\nabla \cdot(\rho \mathbf{v}) & =0 \\
\dot{\mathbf{v}}+(\mathbf{v} \cdot \nabla) \mathbf{v}+\frac{1}{\rho} \nabla p+\nabla \varphi & =0 \\
\nabla^{2} \varphi & =4 \pi G \rho \\
\dot{S}+(\mathbf{v} \cdot \nabla) S & =0 \\
p & =p(\rho, S) .
\end{aligned}
$$

The first equation is the continuity equation, the second is the Euler (force) equation, the third is the Poisson equation of Newtonian gravity, the fourth expresses entropy conservation, and the last describes the equation of state of matter. The derivative with respect to time is denoted by an over-dot.

The background is given by the background energy density $\rho_{o}$, the background pressure $p_{0}$, vanishing velocity, constant gravitational potential $\varphi_{0}$ and constant entropy density $S_{0}$. As mentioned above, it does not satisfy the background Poisson equation.

The equations for cosmological perturbations are obtained by perturbing the fluid variables about the background,

$$
\begin{aligned}
\rho & =\rho_{0}+\delta \rho \\
\mathbf{v} & =\delta \mathbf{v} \\
p & =p_{0}+\delta p \\
\varphi & =\varphi_{0}+\delta \varphi \\
S & =S_{0}+\delta S,
\end{aligned}
$$

where the fluctuating fields $\delta \rho, \delta \mathbf{v}, \delta p, \delta \varphi$ and $\delta S$ are functions of space and time, by inserting these expressions into the basic hydrodynamical 
equations (1.21), by linearizing, and by combining the resulting equations which are of first order in time. We get the following differential equations for the energy density fluctuation $\delta \rho$ and the entropy perturbation $\delta S$

$$
\begin{aligned}
\ddot{\delta} \rho-c_{s}^{2} \nabla^{2} \delta \rho-4 \pi G \rho_{0} \delta \rho & =\sigma \nabla^{2} \delta S \\
\dot{\delta} S & =0,
\end{aligned}
$$

where the variables $c_{s}^{2}$ and $\sigma$ describe the equation of state

$$
\delta p=c_{s}^{2} \delta \rho+\sigma \delta S
$$

with

$$
c_{s}^{2}=\left(\frac{\delta p}{\delta \rho}\right)_{\left.\right|_{S}}
$$

denoting the square of the speed of sound.

Sincethe equations are linear, we can work in Fourier space. Each Fourier component $\delta \rho_{k}(t)$ of the fluctuation field $\delta \rho(\mathbf{x}, t)$

$$
\delta \rho(\mathbf{x}, t)=\int e^{i \mathbf{k} \cdot \mathbf{x}} \delta \rho_{k}(t)
$$

evolves independently.

The fluctuations can be classified as follows: If $\delta S$ vanishes, we have adiabatic fluctuations. If the $\delta S$ is non-vanishing but $\dot{\delta} \rho=0$, we speak of an entropy fluctuation.

The first conclusions we can draw from the basic perturbation equations (1.24) are that

1) entropy fluctuations do not grow,

2) adiabatic fluctuations are time-dependent, and

3) entropy fluctuations seed an adiabatic mode.

Taking a closer look at the equation of motion for $\delta \rho$, we see that the third term on the left hand side represents the force due to gravity, a purely attractive force yielding an instability of flat space-time to the development of density fluctuations (as discussed earlier, see (1.21)). The second term on the left hand side of (1.24) represents a force due to the fluid pressure which tends to set up pressure waves. In the absence of entropy fluctuations, the evolution of $\delta \rho$ is governed by the combined action of both pressure and gravitational forces.

Restricting our attention to adiabatic fluctuations, we see from (1.24) that there is a critical wavelength, the Jeans length, whose wavenumber $k_{J}$ is given by

$$
k_{J}=\left(\frac{4 \pi G \rho_{0}}{c_{s}^{2}}\right)^{1 / 2}
$$


Fluctuations with wavelength longer than the Jeans length $\left(k \ll k_{J}\right)$ grow exponentially

$$
\delta \rho_{k}(t) \sim e^{\omega_{k} t} \text { with } \omega_{k} \sim 4\left(\pi G \rho_{0}\right)^{1 / 2}
$$

whereas short wavelength modes $\left(k \gg k_{J}\right)$ oscillate with frequency $\omega_{k} \sim$ $c_{s} k$. Note that the value of the Jeans length depends on the equation of state of the background. For a background dominated by relativistic radiation, the Jeans length is large (of the order of the Hubble radius $\left.H^{-1}(t)\right)$, whereas for pressure-less matter it goes to zero.

\subsection{PERTURBATIONS ABOUT AN EXPANDING BACKGROUND}

Let us now improve on the previous analysis and study Newtonian cosmological fluctuations about an expanding background. In this case, the background equations are consistent (the non-vanishing average energy density leads to cosmological expansion). However, we are still neglecting general relativistic effects (the fluctuations of the metric). Such effects turn out to be dominant on length scales larger than the Hubble radius $H^{-1}(t)$, and thus the analysis of this section is applicable only to smaller scales.

The background cosmological model is given by the energy density $\rho_{0}(t)$, the pressure $p_{0}(t)$, and the recessional velocity $\mathbf{v}_{0}=H(t) \mathbf{x}$, where $\mathbf{x}$ is the Euclidean spatial coordinate vector ("physical coordinates"). The space- and time-dependent fluctuating fields are defined as in the previous section:

$$
\begin{aligned}
\rho(t, \mathbf{x}) & =\rho_{0}(t)\left(1+\delta_{\epsilon}(t, \mathbf{x})\right) \\
\mathbf{v}(t, \mathbf{x}) & =\mathbf{v}_{0}(t, \mathbf{x})+\delta \mathbf{v}(t, \mathbf{x}) \\
p(t, \mathbf{x}) & =p_{0}(t)+\delta p(t, \mathbf{x}),
\end{aligned}
$$

where $\delta_{\epsilon}$ is the fractional energy density perturbation (we are interested in the fractional rather than in the absolute energy density fluctuation!), and the pressure perturbation $\delta p$ is defined as in (1.25). In addition, there is the possibility of a non-vanishing entropy perturbation defined as in (1.22).

We now insert this ansatz into the basic hydrodynamical equations (1.21), linearize in the perturbation variables, and combine the first order differential equations for $\delta_{\epsilon}$ and $\delta p$ into a single second order differential equation for $\delta \rho_{\epsilon}$. The result simplifies if we work in "comoving coordinates" $\mathbf{q}$ which are the coordinates painted onto the expanding background, i.e.

$$
\mathbf{x}(t)=a(t) \mathbf{q}(t) .
$$


After some algebra, we obtain the following equation which describes the time evolution of density fluctuations:

$$
\ddot{\delta_{\epsilon}}+2 H \dot{\delta_{\epsilon}}-\frac{c_{s}^{2}}{a^{2}} \nabla_{q}^{2} \delta_{\epsilon}-4 \pi G \rho_{0} \delta_{\epsilon}=\frac{\sigma}{\rho_{0} a^{2}} \delta S,
$$

where the subscript $q$ on the $\nabla$ operator indicates that derivatives with respect to comoving coordinates are used. In addition, we have the equation of entropy conservation

$$
\dot{\delta S}=0 .
$$

Comparing with the equations (1.24) obtained in the absence of an expanding background, we see that the only difference is the presence of a Hubble damping term in the equation for $\delta_{\epsilon}$. This term will moderate the exponential instability of the background to long wavelength density fluctuations. In addition, it will lead to a damping of the oscillating solutions on short wavelengths. More specifically, for physical wavenumbers $k_{p} \ll k_{J}$ (where $k_{J}$ is again given by (1.28)), and in a matter-dominated background cosmology, the general solution of (1.32) in the absence of any entropy fluctuations is given by

$$
\delta_{k}(t)=c_{1} t^{2 / 3}+c_{2} t^{-1},
$$

where $c_{1}$ and $c_{2}$ are two constants determined by the initial conditions, and we have dropped the subscript $\epsilon$ in expressions involving $\delta_{\epsilon}$. There are two fundamental solutions, the first is a growing mode with $\delta_{k}(t) \sim$ $a(t)$, the second a decaying mode with $\delta_{k}(t) \sim t^{-1}$. On short wavelength, one obtains damped oscillatory motion:

$$
\delta_{k}(t) \sim a^{-1 / 2}(t) \exp \left( \pm i c_{s} k \int d t^{\prime} a^{-1}\left(t^{\prime}\right)\right) .
$$

As a simple application of the Newtonian equations for cosmological perturbations derived above, let us compare the predicted cosmic microwave background (CMB) anisotropies in a spatially flat Universe with only baryonic matter - Model A - to the corresponding anisotropies in a flat Universe with mostly cold dark matter (pressure-less non-baryonic dark matter) - Model B. We start with the observationally known amplitude of the relative density fluctuations today (time $t_{0}$ ), and we use the fact that the amplitude of the CMB anisotropies on the angular scale $\theta(k)$ corresponding to the comoving wavenumber $k$ is set by the primordial value of the gravitational potential $\phi$ - introduced in the following section - which in turn is related to the primordial value of the density 
fluctuations at Hubble radius crossing (and not to its value of the time $\left.t_{r e c}\right)$. See e.g. Chapter 17 of [36]).

In Model $\mathrm{A}$, the dominant component of the pressure-less matter is coupled to radiation between $t_{e q}$ and $t_{r e c}$, the time of last scattering. Thus, the Jeans length is comparable to the Hubble radius. Therefore, for comoving galactic scales, $k \gg k_{J}$ in this time interval, and thus the fractional density contrast decreases as $a(t)^{-1 / 2}$. In contrast, in Model $\mathrm{B}$, the dominant component of pressure-less matter couples only weakly to radiation, and hence the Jeans length is negligibly small. Thus, in Model $\mathrm{B}$, the relative density contrast grows as $a(t)$ between $t_{e q}$ and $t_{r e c}$. In the time interval $t_{r e c}<t<t_{0}$, the fluctuations scale identically in Models A and B. Summarizing, we conclude, working backwards in time from a fixed amplitude of $\delta_{k}$ today, that the amplitudes of $\delta_{k}\left(t_{e q}\right)$ in Models A and B (and thus their primordial values) are related by

$$
\left.\left.\delta_{k}\left(t_{e q}\right)\right|_{A} \simeq\left(\frac{a\left(t_{r e c}\right)}{a\left(t_{e q}\right)}\right) \delta_{k}\left(t_{e q}\right)\right|_{B} .
$$

Hence, in Model A (without non-baryonic dark matter) the CMB anisotropies are predicted to be a factor of about 30 larger [41] than in Model B, way in excess of the recent observational results. This is one of the strongest arguments for the existence of non-baryonic dark matter. Note that the precise value of the enhancement factor depends on the value of the cosmological constant $\Lambda$ - the above value holds for $\Lambda=0$.

\subsection{CHARACTERIZING PERTURBATIONS}

Let us consider perturbations on a fixed comoving length scale given by a comoving wavenumber $k$. The corresponding physical length increases as $a(t)$. This is to be compared to the Hubble radius $H^{-1}(t)$ which scales as $t$ provided $a(t)$ grows as a power of $t$. In the late time Universe, $a(t) \sim t^{1 / 2}$ in the radiation-dominated phase (i.e. for $t<t_{e q}$, and $a(t) \sim t^{2 / 3}$ in the matter-dominated period $\left(t_{e q}<t<t_{0}\right)$. Thus, we see that at sufficiently early times, all comoving scales had a physical length larger than the Hubble radius. If we consider large cosmological scales (e.g. those corresponding to the observed CMB anisotropies or to galaxy clusters), the time $t_{H}(k)$ of "Hubble radius crossing" (when the physical length was equal to the Hubble radius) was in fact later than $t_{e q}$. As we will see in later sections, the time of Hubble radius crossing plays an important role in the evolution of cosmological perturbations.

Cosmological fluctuations can be described either in position space or in momentum space. In position space, we compute the root mean square mass fluctuation $\delta M / M(k, t)$ in a sphere of radius $l=2 \pi / k$ 
at time $t$. A scale-invariant spectrum of fluctuations is defined by the relation

$$
\frac{\delta M}{M}\left(k, t_{H}(k)\right)=\text { const. . }
$$

Such a spectrum was first suggested by Harrison [42] and Zeldovich [43] as a reasonable choice for the spectrum of cosmological fluctuations. We can introduce the "spectral index" $n$ of cosmological fluctuations by the relation

$$
\left(\frac{\delta M}{M}\right)^{2}\left(k, t_{H}(k)\right) \sim k^{n-1},
$$

and thus a scale-invariant spectrum corresponds to $n=1$.

To make the transition to the (more frequently used) momentum space representation, we Fourier decompose the fractional spatial density contrast

$$
\delta_{\epsilon}(\mathbf{x}, t)=\int d^{3} k \tilde{\delta}_{\epsilon}(\mathbf{k}, t) e^{i \mathbf{k} \cdot \mathbf{x}}
$$

The power spectrum $P_{\delta}$ of density fluctuations is defined by

$$
P_{\delta}(k)=k^{3}\left|\tilde{\delta}_{\epsilon}(k)\right|^{2},
$$

where $k$ is the magnitude of $\mathbf{k}$, and we have assumed for simplicity a Gaussian distribution of fluctuations in which the amplitude of the fluctuations only depends on $k$.

We can also define the power spectrum of the gravitational potential $\varphi$ :

$$
P_{\varphi}(k)=k^{3}|\tilde{\delta \varphi}(k)|^{2} .
$$

These two power spectra are related by the Poisson equation (1.21)

$$
P_{\varphi}(k) \sim k^{-4} P_{\delta}(k) .
$$

In general, the condition of scale-invariance is expressed in momentum space in terms of the power spectrum evaluated at a fixed time. To obtain this condition, we first use the time dependence of the fractional density fluctuation from (1.34) to determine the mass fluctuations at a fixed time $t>t_{H}(k)>t_{e q}$ (the last inequality is a condition on the scales considered)

$$
\left(\frac{\delta M}{M}\right)^{2}(k, t)=\left(\frac{t}{t_{H}(k)}\right)^{4 / 3}\left(\frac{\delta M}{M}\right)^{2}\left(k, t_{H}(k)\right) .
$$

The time of Hubble radius crossing is given by

$$
a\left(t_{H}(k)\right) k^{-1}=2 t_{H}(k),
$$


and thus

$$
t_{H}(k)^{1 / 2} \sim k^{-1} .
$$

Inserting this result into (1.43) making use of (1.38) we find

$$
\left(\frac{\delta M}{M}\right)^{2}(k, t) \sim k^{n+3} .
$$

Since, for reasonable values of the index of the power spectrum, $\delta M / M(k, t)$ is dominated by the Fourier modes with wavenumber $k$, we find that (1.46) implies

$$
\left|\tilde{\delta}_{\epsilon}\right|^{2} \sim k^{n}
$$

or, equivalently,

$$
P_{\varphi}(k) \sim k^{n-1}
$$

\section{RELATIVISTIC THEORY OF COSMOLOGICAL FLUCTUATIONS \\ 4.1 INTRODUCTION}

The Newtonian theory of cosmological fluctuations discussed in the previous section breaks down on scales larger than the Hubble radius because it neglects perturbations of the metric, and because on large scales the metric fluctuations dominate the dynamics.

Let us begin with a heuristic argument to show why metric fluctuations are important on scales larger than the Hubble radius. For such inhomogeneities, one should be able to approximately describe the evolution of the space-time by applying the first FRW equation (1.4) of homogeneous and isotropic cosmology to the local Universe (this approximation is made more rigorous in [44]). Based on this equation, a large-scale fluctuation of the energy density will lead to a fluctuation (" $\delta a ")$ of the scale factor $a$ which grows in time. This is due to the fact that self gravity amplifies fluctuations even on length scales $\lambda$ greater than the Hubble radius.

This argument is made rigorous in the following analysis of cosmological fluctuations in the context of general relativity, where both metric and matter inhomogeneities are taken into account. We will consider fluctuations about a homogeneous and isotropic background cosmology, given by the metric (1.1), which can be written in conformal time $\eta$ (defined by $d t=a(t) d \eta$ ) as

$$
d s^{2}=a(\eta)^{2}\left(d \eta^{2}-d \mathbf{x}^{2}\right) .
$$


The theory of cosmological perturbations is based on expanding the Einstein equations to linear order about the background metric. The theory was initially developed in pioneering works by Lifshitz [45]. Significant progress in the understanding of the physics of cosmological fluctuations was achieved by Bardeen [46] who realized the importance of subtracting gauge artifacts (see below) from the analysis (see also [47]). The following discussion is based on Part I of the comprehensive review article [36]. Other reviews - in some cases emphasizing different approaches - are [48, 49, 50, 51].

\subsection{CLASSIFYING FLUCTUATIONS}

The first step in the analysis of metric fluctuations is to classify them according to their transformation properties under spatial rotations. There are scalar, vector and second rank tensor fluctuations. In linear theory, there is no coupling between the different fluctuation modes, and hence they evolve independently (for some subtleties in this classification, see [52]).

We begin by expanding the metric about the FRW background metric $g_{\mu \nu}^{(0)}$ given by (1.49):

$$
g_{\mu \nu}=g_{\mu \nu}^{(0)}+\delta g_{\mu \nu}
$$

The background metric depends only on time, whereas the metric fluctuations $\delta g_{\mu \nu}$ depend on both space and time. Since the metric is a symmetric tensor, there are at first sight 10 fluctuating degrees of freedom in $\delta g_{\mu \nu}$.

There are four degrees of freedom which correspond to scalar metric fluctuations (the only four ways of constructing a metric from scalar functions):

$$
\delta g_{\mu \nu}=a^{2}\left(\begin{array}{cc}
2 \phi & -B_{, i} \\
-B_{, i} & 2\left(\psi \delta_{i j}-E_{, i j}\right)
\end{array}\right)
$$

where the four fluctuating degrees of freedom are denoted (following the notation of [36]) $\phi, B, E$, and $\psi$, a comma denotes the ordinary partial derivative (if we had included spatial curvature of the background metric, it would have been the covariant derivative with respect to the spatial metric), and $\delta_{i j}$ is the Kronecker symbol.

There are also four vector degrees of freedom of metric fluctuations, consisting of the four ways of constructing metric fluctuations from three vectors:

$$
\delta g_{\mu \nu}=a^{2}\left(\begin{array}{cc}
0 & -S_{i} \\
-S_{i} & F_{i, j}+F_{j, i}
\end{array}\right)
$$


where $S_{i}$ and $F_{i}$ are two divergence-less vectors (for a vector with nonvanishing divergence, the divergence contributes to the scalar gravitational fluctuation modes).

Finally, there are two tensor modes which correspond to the two polarization states of gravitational waves:

$$
\delta g_{\mu \nu}=-a^{2}\left(\begin{array}{cc}
0 & 0 \\
0 & h_{i j}
\end{array}\right)
$$

where $h_{i j}$ is trace-free and divergence-less

$$
h_{i}^{i}=h_{i j}^{j}=0 .
$$

Gravitational waves do not couple at linear order to the matter fluctuations. Vector fluctuations decay in an expanding background cosmology and hence are not usually cosmologically important. The most important fluctuations, at least in inflationary cosmology, are the scalar metric fluctuations, the fluctuations which couple to matter inhomogeneities and which are the relativistic generalization of the Newtonian perturbations considered in the previous section.

\subsection{GAUGE TRANSFORMATION}

The theory of cosmological perturbations is at first sight complicated by the issue of gauge invariance (at the final stage, however, we will see that we can make use of the gauge freedom to substantially simplify the theory). The coordinates $t, \mathbf{x}$ of space-time carry no independent physical meaning. They are just labels to designate points in the spacetime manifold. By performing a small-amplitude transformation of the space-time coordinates (called "gauge transformation" in the following), we can easily introduce "fictitious" fluctuations in a homogeneous and isotropic Universe. These modes are "gauge artifacts".

We will in the following take an "active" view of gauge transformation. Let us consider two space-time manifolds, one of them a homogeneous and isotropic Universe $\mathcal{M}_{0}$, the other a physical Universe $\mathcal{M}$ with inhomogeneities. A choice of coordinates can be considered to be a mapping $\mathcal{D}$ between the manifolds $\mathcal{M}_{0}$ and $\mathcal{M}$. Let us consider a second mapping $\tilde{\mathcal{D}}$ which will map the same point (e.g. the origin of a fixed coordinate system) in $\mathcal{M}_{0}$ into different points in $\mathcal{M}$. Using the inverse of these maps $\mathcal{D}$ and $\tilde{\mathcal{D}}$, we can assign two different sets of coordinates to points in $\mathcal{M}$.

Consider now a physical quantity $Q$ (e.g. the Ricci scalar) on $\mathcal{M}$, and the corresponding physical quantity $Q^{(0)}$ on $\mathcal{M}_{0}$ Then, in the first coordinate system given by the mapping $\mathcal{D}$, the perturbation $\delta Q$ of $Q$ 
at the point $p \in \mathcal{M}$ is defined by

$$
\delta Q(p)=Q(p)-Q^{(0)}\left(\mathcal{D}^{-1}(p)\right) .
$$

Analogously, in the second coordinate system given by $\tilde{\mathcal{D}}$, the perturbation is defined by

$$
\tilde{\delta Q}(p)=Q(p)-Q^{(0)}\left(\tilde{\mathcal{D}}^{-1}(p)\right)
$$

The difference

$$
\Delta Q(p)=\tilde{\delta Q}(p)-\delta Q(p)
$$

is obviously a gauge artifact and carries no physical significance.

Some of the metric perturbation degrees of freedom introduced in the first subsection are gauge artifacts. To isolate these, we must study how coordinate transformations act on the metric. There are four independent gauge degrees of freedom corresponding to the coordinate transformation

$$
x^{\mu} \rightarrow \tilde{x}^{\mu}=x^{\mu}+\xi^{\mu} .
$$

The zero (time) component $\xi^{0}$ of $\xi^{\mu}$ leads to a scalar metric fluctuation. The spatial three vector $\xi^{i}$ can be decomposed as

$$
\xi^{i}=\xi_{t r}^{i}+\gamma^{i j} \xi_{, j}
$$

(where $\gamma^{i j}$ is the spatial background metric) into a transverse piece $\xi_{t r}^{i}$ which has two degrees of freedom which yield vector perturbations, and the second term (given by the gradient of a scalar $\xi$ ) which gives a scalar fluctuation. To summarize this paragraph, there are two scalar gauge modes given by $\xi^{0}$ and $\xi$, and two vector modes given by the transverse three vector $\xi_{t r}^{i}$. Thus, there remain two physical scalar and two vector fluctuation modes. The gravitational waves are gauge-invariant.

Let us now focus on how the scalar gauge transformations (i.e. the transformations given by $\xi^{0}$ and $\xi$ ) act on the scalar metric fluctuation variables $\phi, B, E$, and $\psi$. An immediate calculation yields:

$$
\begin{aligned}
\tilde{\phi} & =\phi-\frac{a^{\prime}}{a} \xi^{0}-\left(\xi^{0}\right)^{\prime} \\
\tilde{B} & =B+\xi^{0}-\xi^{\prime} \\
\tilde{E} & =E-\xi \\
\tilde{\psi} & =\psi+\frac{a^{\prime}}{a} \xi^{0},
\end{aligned}
$$

where a prime indicates the derivative with respect to conformal time $\eta$.

There are two approaches to deal with the gauge ambiguities. The first is to fix a gauge, i.e. to pick conditions on the coordinates which 
completely eliminate the gauge freedom, the second is to work with a basis of gauge-invariant variables.

If one wants to adopt the gauge-fixed approach, there are many different gauge choices. Note that the often used synchronous gauge determined by $\delta g^{0 \mu}=0$ does not totally fix the gauge. A convenient system which completely fixes the coordinates is the so-called longitudinal or conformal Newtonian gauge defined by $B=E=0$.

If one prefers a gauge-invariant approach, there are many choices of gauge-invariant variables. A convenient basis first introduced by [46] is the basis $\Phi, \Psi$ given by

$$
\begin{aligned}
\Phi & =\phi+\frac{1}{a}\left[\left(B-E^{\prime}\right) a\right]^{\prime} \\
\Psi & =\psi-\frac{a^{\prime}}{a}\left(B-E^{\prime}\right) .
\end{aligned}
$$

It is obvious from the above equations that the gauge-invariant variables $\Phi$ and $\Psi$ coincide with the corresponding diagonal metric perturbations $\phi$ and $\psi$ in longitudinal gauge.

Note that the variables defined above are gauge-invariant only under linear space-time coordinate transformations. Beyond linear order, the structure of perturbation theory becomes much more involved. In fact, one can show [53] that the only fluctuation variables which are invariant under all coordinate transformations are perturbations of variables which are constant in the background space-time.

\subsection{EQUATIONS OF MOTION}

We begin with the Einstein equations

$$
G_{\mu \nu}=8 \pi G T_{\mu \nu}
$$

where $G_{\mu \nu}$ is the Einstein tensor associated with the space-time metric $g_{\mu \nu}$, and $T_{\mu \nu}$ is the energy-momentum tensor of matter, insert the ansatz for metric and matter perturbed about a FRW background $\left(g_{\mu \nu}^{(0)}(\eta), \varphi^{(0)}(\eta)\right)$ :

$$
\begin{aligned}
g_{\mu \nu}(\mathbf{x}, \eta) & =g_{\mu \nu}^{(0)}(\eta)+\delta g_{\mu \nu}(\mathbf{x}, \eta) \\
\varphi(\mathbf{x}, \eta) & =\varphi_{0}(\eta)+\delta \varphi(\mathbf{x}, \eta)
\end{aligned}
$$

(where we have for simplicity replaced general matter by a scalar matter field $\varphi$ ) and expand to linear order in the fluctuating fields, obtaining the following equations:

$$
\delta G_{\mu \nu}=8 \pi G \delta T_{\mu \nu} .
$$


In the above, $\delta g_{\mu \nu}$ is the perturbation in the metric and $\delta \varphi$ is the fluctuation of the matter field $\varphi$.

Note that the components $\delta G_{\nu}^{\mu}$ and $\delta T_{\nu}^{\mu}$ are not gauge invariant. If we want to use the gauge-invariant approach, we note [36] that it is possible to construct a gauge-invariant tensor $\delta G_{\nu}^{(g i) \mu}$ via

$$
\begin{aligned}
\delta G_{0}^{(g i) 0} & \equiv \delta G_{0}^{0}+\left({ }^{(0)} G_{0}^{\prime}{ }^{0}\right)\left(B-E^{\prime}\right) \\
\delta G_{i}^{(g i) 0} & \equiv \delta G_{i}^{0}+\left({ }^{(0)} G_{i}^{0}-\frac{1}{3}{ }^{(0)} G_{k}^{k}\right)\left(B-E^{\prime}\right)_{, i} \\
\delta G_{j}^{(g i) i} & \equiv \delta G_{j}^{i}+\left({ }^{(0)} G_{j}^{\prime}{ }^{i}\right)\left(B-E^{\prime}\right),
\end{aligned}
$$

where $(0) G_{\nu}^{\mu}$ denote the background values of the Einstein tensor. Analogously, a gauge-invariant linearized stress-energy tensor $\delta T_{\nu}^{(g i) \mu}$ can be defined. In terms of these tensors, the gauge-invariant form of the equations of motion for linear fluctuations reads

$$
\delta G_{\mu \nu}^{(g i)}=8 \pi G \delta T_{\mu \nu}^{(g i)} .
$$

If we insert into this equation the ansatz for the general metric and matter fluctuations (which depend on the gauge), only gauge-invariant combinations of the fluctuation variables will appear.

In a gauge-fixed approach, one can start with the metric in longitudinal gauge

$$
d s^{2}=a^{2}\left[(1+2 \phi) d \eta^{2}-(1-2 \psi) \gamma_{i j} d x^{i} d x^{j}\right]
$$

and insert this ansatz into the general perturbation equations (1.66). The shortcut of inserting a restricted ansatz for the metric into the action and deriving the full set of variational equations is justified in this case.

Both approaches yield the following set of equations of motion:

$$
\begin{aligned}
-3 \mathcal{H}\left(\mathcal{H} \Phi+\Psi^{\prime}\right)+\nabla^{2} \Psi & =4 \pi G a^{2} \delta T_{0}^{(g i) 0} \\
\left(\mathcal{H} \Phi+\Psi^{\prime}\right)_{, i} & =4 \pi G a^{2} \delta T_{i}^{(g i) 0} \\
{\left[\left(2 \mathcal{H}^{\prime}+\mathcal{H}^{2}\right) \Phi+\mathcal{H} \Phi^{\prime}+\Psi^{\prime \prime}+2 \mathcal{H} \Psi^{\prime}\right] \delta_{j}^{i} } & \\
+\frac{1}{2} \nabla^{2} D \delta_{j}^{i}-\frac{1}{2} \gamma^{i k} D_{, k j} & =-4 \pi G a^{2} \delta T_{j}^{(g i) i},
\end{aligned}
$$

where $D \equiv \Phi-\Psi$ and $\mathcal{H}=a^{\prime} / a$. If we work in longitudinal gauge, then $\delta T_{j}^{(g i) i}=\delta T_{j}^{i}, \Phi=\phi$ and $\Psi=\psi$.

The first conclusion we can draw is that if no anisotropic stress is present in the matter at linear order in fluctuating fields, i.e. $\delta T_{j}^{i}=0$ for $i \neq j$, then the two metric fluctuation variables coincide:

$$
\Phi=\Psi .
$$


This will be the case in most simple cosmological models, e.g. in theories with matter described by a set of scalar fields with canonical form of the action, and in the case of a perfect fluid with no anisotropic stress.

Let us now restrict our attention to the case of matter described in terms of a single scalar field $\varphi$ which can be expanded as

$$
\varphi(\mathbf{x}, \eta)=\varphi_{0}(\eta)+\delta \varphi(\mathbf{x}, \eta)
$$

in terms of background matter $\varphi_{0}$ and matter fluctuation $\delta \varphi(\mathbf{x}, \eta)$. Then, in longitudinal gauge, (1.69) reduce to the following set of equations of motion (making use of (1.71))

$$
\begin{aligned}
\nabla^{2} \phi-3 \mathcal{H} \phi^{\prime}-\left(\mathcal{H}^{\prime}+2 \mathcal{H}^{2}\right) \phi & =4 \pi G\left(\varphi_{0}^{\prime} \delta \varphi^{\prime}+V^{\prime} a^{2} \delta \varphi\right) \\
\phi^{\prime}+\mathcal{H} \phi & =4 \pi G \varphi_{0}^{\prime} \delta \varphi \\
\phi^{\prime \prime}+3 \mathcal{H} \phi^{\prime}+\left(\mathcal{H}^{\prime}+2 \mathcal{H}^{2}\right) \phi & =4 \pi G\left(\varphi_{0}^{\prime} \delta \varphi^{\prime}-V^{\prime} a^{2} \delta \varphi\right),
\end{aligned}
$$

where $V^{\prime}$ denotes the derivative of $V$ with respect to $\varphi$. These equations can be combined to give the following second order differential equation for the relativistic potential $\phi$ :

$$
\phi^{\prime \prime}+2\left(\mathcal{H}-\frac{\varphi_{0}^{\prime \prime}}{\varphi_{0}^{\prime}}\right) \phi^{\prime}-\nabla^{2} \phi+2\left(\mathcal{H}^{\prime}-\mathcal{H} \frac{\varphi_{0}^{\prime \prime}}{\varphi_{0}^{\prime}}\right) \phi=0 .
$$

This is the final result for the classical evolution of cosmological fluctuations. First of all, we note the similarities with the equation (1.32) obtained in the Newtonian theory. The final term in (1.74) is the force due to gravity leading to the instability, the second to last term is the pressure force leading to oscillations (relativistic since we are considering matter to be a relativistic field), and the second term is the Hubble friction term. For each wavenumber there are two fundamental solutions. On small scales $(k>H)$, the solutions correspond to damped oscillations, on large scales $(k<H)$ the oscillations freeze out and the dynamics is governed by the gravitational force competing with the Hubble friction term. Note, in particular, how the Hubble radius naturally emerges as the scale where the nature of the fluctuating modes changes from oscillatory to frozen.

Considering the equation in a bit more detail, observe that if the equation of state of the background is independent of time (which will be the case if $\mathcal{H}^{\prime}=\varphi_{0}^{\prime \prime}=0$ ), then in an expanding background, the dominant mode of (1.74) is constant, and the sub-dominant mode decays. If the equation of state is not constant, then the dominant mode is not constant in time. Specifically, at the end of inflation $\mathcal{H}^{\prime}<0$, and this leads to a growth of $\phi$ (see the following subsection). 
To study the quantitative implications of the equation of motion (1.74), it is convenient to introduce $[54,55]$ the variable $\zeta$ (which, up to correction term of the order $\nabla^{2} \phi$ which is unimportant for large-scale fluctuations, is equal to the curvature perturbation $\mathcal{R}$ in comoving gauge [56]) by

$$
\zeta \equiv \phi+\frac{2}{3} \frac{\left(H^{-1} \dot{\phi}+\phi\right)}{1+w}
$$

where

$$
w=\frac{p}{\rho}
$$

characterizes the equation of state of matter. In terms of $\zeta$, the equation of motion (1.74) takes on the form

$$
\frac{3}{2} \dot{\zeta} H(1+w)=\mathcal{O}\left(\nabla^{2} \phi\right) .
$$

On large scales, the right hand side of the equation is negligible, which leads to the conclusion that large-scale cosmological fluctuations satisfy

$$
\dot{\zeta}(1+w)=0 .
$$

This implies that $\zeta$ is constant except possibly if $1+w=0$ at some point in time during the cosmological evolution (which occurs during reheating in inflationary cosmology if the inflaton field undergoes oscillations - see $[57]$ and $[58,59]$ for discussions of the consequences in single and double field inflationary models, respectively). In single matter field models it is indeed possible to show that $\dot{\zeta}=0$ on super-Hubble scales independent of assumptions on the equation of state [66, 67]. This "conservation law" makes it easy to relate initial fluctuations to final fluctuations in inflationary cosmology, as will be illustrated in the following subsection.

\subsection{APPLICATION TO INFLATIONARY COSMOLOGY}

Let us now return to the space-time sketch of the evolution of fluctuations in inflationary cosmology - see Figure (1.1) - and use the conservation law (1.78) - in the form $\zeta=$ const on large scales - to relate the amplitude of $\phi$ at initial Hubble radius crossing during the inflationary phase (at $\left.t=t_{i}(k)\right)$ with the amplitude at final Hubble radius crossing at late times (at $t=t_{f}(k)$ ). Since both at early times and at late times $\dot{\phi}=0$ on super-Hubble scales as the equation of state is not changing, (1.78) and (1.75) lead to

$$
\phi\left(t_{f}(k)\right) \simeq \frac{(1+w)\left(t_{f}(k)\right)}{(1+w)\left(t_{i}(k)\right)} \phi\left(t_{i}(k)\right) .
$$


This equation will allow us to evaluate the amplitude of the cosmological perturbations when they re-enter the Hubble radius at time $t_{f}(k)$, under the assumption (discussed in detail in the following section) that the origin of the primordial fluctuations is quantum vacuum oscillations.

The time-time perturbed Einstein equation (the first equation of (1.69)) relates the value of $\phi$ at initial Hubble radius crossing to the amplitude of the fractional energy density fluctuations. This, together with the fact that the amplitude of the scalar matter field quantum vacuum fluctuations is of the order $H$, yields

$$
\phi\left(t_{i}(k)\right) \sim H \frac{V^{\prime}}{V}\left(t_{i}(k)\right) .
$$

In the late time radiation dominated phase, $w=1 / 3$, whereas during slow-roll inflation

$$
1+w\left(t_{i}(k)\right) \simeq \frac{\dot{\varphi}_{0}^{2}}{V}\left(t_{i}(k)\right) .
$$

Making, in addition, use of the slow roll conditions satisfied during the inflationary period

$$
\begin{aligned}
H \dot{\varphi}_{0} & \simeq-V^{\prime} \\
H^{2} & \simeq \frac{8 \pi G}{3} V,
\end{aligned}
$$

we arrive at the final result

$$
\phi\left(t_{f}(k)\right) \sim \frac{V^{3 / 2}}{V^{\prime} m_{p l}^{3}}\left(t_{i}(k)\right),
$$

which gives the position space amplitude of cosmological fluctuations on a scale labelled by the comoving wavenumber $k$ at the time when the scale re-enters the Hubble radius at late times, a result first obtained in the case of the Starobinsky model [14] of inflation in [68], and later in the context of scalar field-driven inflation in $[69,70,71,54]$.

In the case of slow roll inflation, the right hand side of (1.83) is, to a first approximation, independent of $k$, and hence the resulting spectrum of fluctuations is nearly scale-invariant.

\section{QUANTUM THEORY OF COSMOLOGICAL FLUCTUATIONS}

\subsection{OVERVIEW}

As already mentioned in the previous section, in many models of the very early Universe, in particular in inflationary cosmology, but also 
in the Pre-Big-Bang and in the Ekpyrotic scenarios, primordial inhomogeneities emerge from quantum vacuum fluctuations on microscopic scales (wavelengths smaller than the Hubble radius). The wavelength is then stretched relative to the Hubble radius, becomes larger than the Hubble radius at some time and then propagates on super-Hubble scales until re-entering at late cosmological times. In the context of a Universe with a de Sitter phase, the quantum origin of cosmological fluctuations was first discussed in [68] - see [72] for a more general discussion of the quantum origin of fluctuations in cosmology, and also [73, 74] for earlier ideas. In particular, Mukhanov [68] and Press [73] realized that in an exponentially expanding background, the curvature fluctuations would be scale-invariant, and Mukhanov provided a quantitative calculation which also yielded the logarithmic deviation from exact scale-invariance.

To understand the role of the Hubble radius, consider the equation of a free scalar matter field $\varphi$ on an unperturbed expanding background:

$$
\ddot{\varphi}+3 H \dot{\varphi}-\frac{\nabla^{2}}{a^{2}} \varphi=0
$$

The second term on the left hand side of this equation leads to damping of $\varphi$ with a characteristic decay rate given by $H$. As a consequence, in the absence of the spatial gradient term, $\dot{\varphi}$ would be of the order of magnitude $H \varphi$. Thus, comparing the second and the third term on the left hand side, we immediately see that the microscopic (spatial gradient) term dominates on length scales smaller than the Hubble radius, leading to oscillatory motion, whereas this term is negligible on scales larger than the Hubble radius, and the evolution of $\varphi$ is determined primarily by gravity. Note that in general cosmological models the Hubble radius is much smaller than the horizon (the forward light cone calculated from the initial time). In an inflationary universe, the horizon is larger by a factor of at least $\exp (N)$, where $N$ is the number of e-foldings of inflation, and the lower bound is taken on if the Hubble radius and horizon coincide until inflation begins. It is very important to realize this difference, a difference which is obscured in most articles on cosmology in which the term "horizon" is used when "Hubble radius" is meant. Note, in particular, that the homogeneous inflaton field contains causal information on super-Hubble but sub-horizon scales. Hence, it is completely consistent with causality [57] to have a microphysical process related to the background scalar matter field lead to exponential amplification of the amplitude of fluctuations during reheating on such scales, as it does in models in which entropy perturbations are present and not suppressed during inflation $[58,59]$. 
There are, however, general relativistic conservation laws [60] which imply that adiabatic fluctuations produced locally must be Poissonstatistic suppressed on scales larger than the Hubble radius. For example, fluctuations produced by the formation of topological defects at a phase transition in the early universe are initially isocurvature (entropy) in nature (see e.g. [61] for a discussion). Via the source term in the equation of motion (1.24), a growing adiabatic mode is induced, but at any fixed time the spectrum of the curvature fluctuation on scales larger than the Hubble radius has index $n=4$ (Poisson). A similar conclusion applies to recently discussed models $[62,63]$ of modulated reheating as a new source of density perturbations (see [64] for a nice discussion), and to models in which moduli fields obtain masses after some symmetry breaking, their quantum fluctuations then inducing cosmological fluctuations. A prototypical example is given by axion fluctuations in an inflationary universe (see e.g. [65] and references therein).

To understand the generation and evolution of fluctuations in current models of the very early Universe, we need both Quantum Mechanics and General Relativity, i.e. quantum gravity. At first sight, we are thus faced with an intractable problem, since the theory of quantum gravity is not yet established. We are saved by the fact that today on large cosmological scales the fractional amplitude of the fluctuations is smaller than 1. Since gravity is a purely attractive force, the fluctuations had to have been - at least in the context of an eternally expanding background cosmology - very small in the early Universe. Thus, a linearized analysis of the fluctuations (about a classical cosmological background) is selfconsistent.

From the classical theory of cosmological perturbations discussed in the previous section, we know that the analysis of scalar metric inhomogeneities can be reduced - after extracting gauge artifacts - to the study of the evolution of a single fluctuating variable. Thus, we conclude that the quantum theory of cosmological perturbations must be reducible to the quantum theory of a single free scalar field which we will denote by $v$. Since the background in which this scalar field evolves is time-dependent, the mass of $v$ will be time-dependent. The timedependence of the mass will lead to quantum particle production over time if we start the evolution in the vacuum state for $v$. As we will see, this quantum particle production corresponds to the development and growth of the cosmological fluctuations. Thus, the quantum theory of cosmological fluctuations provides a consistent framework to study both the generation and the evolution of metric perturbations. The following analysis is based on Part II of [36]. 


\subsection{OUTLINE OF THE ANALYSIS}

In order to obtain the action for linearized cosmological perturbations, we expand the action to quadratic order in the fluctuating degrees of freedom. The linear terms cancel because the background is taken to satisfy the background equations of motion.

We begin with the Einstein-Hilbert action for gravity and the action of a scalar matter field (for the more complicated case of general hydrodynamical fluctuations the reader is referred to [36])

$$
S=\int d^{4} x \sqrt{-g}\left[-\frac{1}{16 \pi G} R+\frac{1}{2} \partial_{\mu} \varphi \partial^{\mu} \varphi-V(\varphi)\right],
$$

where $R$ is the Ricci curvature scalar.

The simplest way to proceed is to work in longitudinal gauge, in which the metric and matter take the form

$$
\begin{aligned}
d s^{2} & =a^{2}(\eta)\left[(1+2 \phi(\eta, \mathbf{x})) d \eta^{2}-(1-2 \psi(t, \mathbf{x})) d \mathbf{x}^{2}\right] \\
\varphi(\eta, \mathbf{x}) & =\varphi_{0}(\eta)+\delta \varphi(\eta, \mathbf{x}) .
\end{aligned}
$$

The next step is to reduce the number of degrees of freedom. First, as already mentioned in the previous section, the off-diagonal spatial Einstein equations force $\psi=\phi$ since $\delta T_{j}^{i}=0$ for scalar field matter (no anisotropic stresses to linear order). The two remaining fluctuating variables $\phi$ and $\varphi$ must be linked by the Einstein constraint equations since there cannot be matter fluctuations without induced metric fluctuations.

The two nontrivial tasks of the lengthy [36] computation of the quadratic piece of the action is to find out what combination of $\varphi$ and $\phi$ gives the variable $v$ in terms of which the action has canonical kinetic term, and what the form of the time-dependent mass is. This calculation involves inserting the ansatz (1.86) into the action (1.85), expanding the result to second order in the fluctuating fields, making use of the background and of the constraint equations, and dropping total derivative terms from the action. In the context of scalar field matter, the quantum theory of cosmological fluctuations was developed by Mukhanov [75, 76] (see also [77]). The result is the following contribution $S^{(2)}$ to the action quadratic in the perturbations:

$$
S^{(2)}=\frac{1}{2} \int d^{4} x\left[v^{\prime 2}-v_{, i} v_{, i}+\frac{z^{\prime \prime}}{z} v^{2}\right],
$$

where the canonical variable $v$ (the "Mukhanov variable" introduced in [76] - see also [72]) is given by

$$
v=a\left[\delta \varphi+\frac{\varphi_{0}^{\prime}}{\mathcal{H}} \phi\right],
$$


with $\mathcal{H}=a^{\prime} / a$, and where

$$
z=\frac{a \varphi_{0}^{\prime}}{\mathcal{H}}
$$

In both the cases of power law inflation and slow roll inflation, $\mathcal{H}$ and $\varphi_{0}^{\prime}$ are proportional and hence (as long as the equation of state does not change over time)

$$
z(\eta) \sim a(\eta) .
$$

Note that the variable $v$ is related to the curvature perturbation $\mathcal{R}$ in comoving coordinates introduced in [56] and closely related to the variable $\zeta$ used in $[54,55]$ :

$$
v=z \mathcal{R} \text {. }
$$

The equation of motion which follows from the action (1.87) is

$$
v^{\prime \prime}-\nabla^{2} v-\frac{z^{\prime \prime}}{z} v=0
$$

or, in momentum space,

$$
v_{k}^{\prime \prime}+k^{2} v_{k}-\frac{z^{\prime \prime}}{z} v_{k}=0,
$$

where $v_{k}$ is the k'th Fourier mode of $v$. As a consequence of (1.90), the mass term in the above equation is given by the Hubble scale

$$
k_{H}^{2} \equiv \frac{z^{\prime \prime}}{z} \simeq H^{2}
$$

Thus, it immediately follows from (1.93) that on small length scales, i.e. for $k>k_{H}$, the solutions for $v_{k}$ are constant amplitude oscillations . These oscillations freeze out at Hubble radius crossing, i.e. when $k=k_{H}$. On longer scales $\left(k \ll k_{H}\right)$, the solutions for $v_{k}$ increase as $z$ :

$$
v_{k} \sim z, k \ll k_{H} .
$$

Given the action (1.87), the quantization of the cosmological perturbations can be performed by canonical quantization (in the same way that a scalar matter field on a fixed cosmological background is quantized [78]).

The final step in the quantum theory of cosmological perturbations is to specify an initial state. Since in inflationary cosmology all preexisting classical fluctuations are red-shifted by the accelerated expansion of space, one usually assumes (we will return to a criticism of this 
point when discussing the trans-Planckian problem of inflationary cosmology) that the field $v$ starts out at the initial time $t_{i}$ mode by mode in its vacuum state. Two questions immediately emerge: what is the initial time $t_{i}$, and which of the many possible vacuum states should be chosen. It is usually assumed that since the fluctuations only oscillate on sub-Hubble scales, the choice of the initial time is not important, as long as it is earlier than the time when scales of cosmological interest today cross the Hubble radius during the inflationary phase. The state is usually taken to be the Bunch-Davies vacuum (see e.g. [78]), since this state is empty of particles at $t_{i}$ in the coordinate frame determined by the FRW coordinates (see e.g. [79] for a discussion of this point), and since the Bunch-Davies state is a local attractor in the space of initial states in an expanding background (see e.g. [80]). Thus, we choose the initial conditions

$$
\begin{aligned}
& v_{k}\left(\eta_{i}\right)=\frac{1}{\sqrt{2 \omega_{k}}} \\
& v_{k}^{\prime}\left(\eta_{i}\right)=\frac{\sqrt{\omega_{k}}}{\sqrt{2}}
\end{aligned}
$$

where here $\omega_{k}=k$, and $\eta_{i}$ is the conformal time corresponding to the physical time $t_{i}$.

Let us briefly summarize the quantum theory of cosmological perturbations. In the linearized theory, fluctuations are set up at some initial time $t_{i}$ mode by mode in their vacuum state. While the wavelength is smaller than the Hubble radius, the state undergoes quantum vacuum fluctuations. The accelerated expansion of the background redshifts the length scale beyond the Hubble radius. The fluctuations freeze out when the length scale is equal to the Hubble radius. On larger scales, the amplitude of $v_{k}$ increases as the scale factor. This corresponds to the squeezing of the quantum state present at Hubble radius crossing (in terms of classical general relativity, it is self-gravity which leads to this growth of fluctuations). As discussed e.g. in [81], the squeezing of the quantum vacuum state sets up the classical correlations in the wave function of the fluctuations which are an essential ingredient in the classicalization of the perturbations.

\subsection{APPLICATION TO INFLATIONARY COSMOLOGY}

We will now use the quantum theory of cosmological perturbations developed above to calculate the spectrum of curvature fluctuations in inflationary cosmology. 
We need to compute the power spectrum $\mathcal{P}_{\mathcal{R}}(k)$ of the curvature fluctuation $\mathcal{R}$ defined in (1.91), namely

$$
\mathcal{R}=z^{-1} v=\phi+\delta \varphi \frac{\mathcal{H}}{\varphi_{0}^{\prime}}
$$

The idea in calculating the power spectrum at a late time $t$ is to first relate the power spectrum via the growth rate (1.95) of $v$ on superHubble scales to the power spectrum at the time $t_{H}(k)$ of Hubble radius crossing, and to then use the constancy of the amplitude of $v$ on subHubble scales to relate it to the initial conditions (1.96). Thus

$$
\begin{aligned}
\mathcal{P}_{\mathcal{R}}(k, t) \equiv k^{3} \mathcal{R}_{k}^{2}(t) & =k^{3} z^{-2}(t)\left|v_{k}(t)\right|^{2} \\
& =k^{3} z^{-2}(t)\left(\frac{z(t)}{z\left(t_{H}(k)\right)}\right)^{2}\left|v_{k}\left(t_{H}(k)\right)\right|^{2} \\
& =k^{3} z^{-2}\left(t_{H}(k)\right)\left|v_{k}\left(t_{H}(k)\right)\right|^{2} \\
& \sim k^{3} a^{-2}\left(t_{H}(k)\right)\left|v_{k}\left(t_{i}\right)\right|^{2}
\end{aligned}
$$

where in the final step we have used (1.90) and the constancy of the amplitude of $v$ on sub-Hubble scales. Making use of the condition

$$
a^{-1}\left(t_{H}(k)\right) k=H
$$

for Hubble radius crossing, and of the initial conditions (1.96), we immediately see that

$$
\mathcal{P}_{\mathcal{R}}(k, t) \sim k^{3} k^{-2} k^{-1} H^{2},
$$

and that thus a scale invariant power spectrum with amplitude proportional to $H^{2}$ results, in agreement with what was argued on heuristic grounds in Section (4.5).

\subsection{QUANTUM THEORY OF GRAVITATIONAL WAVES}

The quantization of gravitational waves parallels the quantization of scalar metric fluctuations, but is more simple because there are no gauge ambiguities. Note that at the level of linear fluctuations, scalar metric fluctuations and gravitational waves are independent. Both can be quantized on the same cosmological background determined by the background scale factor and the background matter. However, in contrast to the case of scalar metric fluctuations, the tensor modes are also present in pure gravity (i.e. in the absence of matter).

Starting point is the action (1.85). Into this action we insert the metric which corresponds to a classical cosmological background plus 
tensor metric fluctuations:

$$
d s^{2}=a^{2}(\eta)\left[d \eta^{2}-\left(\delta_{i j}+h_{i j}\right) d x^{i} d x^{j}\right],
$$

where the second rank tensor $h_{i j}(\eta, \mathbf{x})$ represents the gravitational waves, and in turn can be decomposed as

$$
h_{i j}(\eta, \mathbf{x})=h_{+}(\eta, \mathbf{x}) e_{i j}^{+}+h_{x}(\eta, \mathbf{x}) e_{i j}^{x}
$$

into the two polarization states. Here, $e_{i j}^{+}$and $e_{i j}^{x}$ are two fixed polarization tensors, and $h_{+}$and $h_{x}$ are the two coefficient functions.

To quadratic order in the fluctuating fields, the action consists of separate terms involving $h_{+}$and $h_{x}$. Each term is of the form

$$
S^{(2)}=\int d^{4} x \frac{a^{2}}{2}\left[h^{\prime 2}-(\nabla h)^{2}\right],
$$

leading to the equation of motion

$$
h_{k}^{\prime \prime}+2 \frac{a^{\prime}}{a} h_{k}^{\prime}+k^{2} h_{k}=0 .
$$

The variable in terms of which the action (1.103) has canonical kinetic term is

$$
\mu_{k} \equiv a h_{k}
$$

and its equation of motion is

$$
\mu_{k}^{\prime \prime}+\left(k^{2}-\frac{a^{\prime \prime}}{a}\right) \mu_{k}=0
$$

This equation is very similar to the corresponding equation (1.93) for scalar gravitational inhomogeneities, except that in the mass term the scale factor $a(\eta)$ replaces $z(\eta)$, which leads to a very different evolution of scalar and tensor modes during the reheating phase in inflationary cosmology during which the equation of state of the background matter changes dramatically.

Based on the above discussion we have the following theory for the generation and evolution of gravitational waves in an accelerating Universe (first developed by Grishchuk [82]): waves exist as quantum vacuum fluctuations at the initial time on all scales. They oscillate until the length scale crosses the Hubble radius. At that point, the oscillations freeze out and the quantum state of gravitational waves begins to be squeezed in the sense that

$$
\mu_{k}(\eta) \sim a(\eta)
$$

which, from (1.105) corresponds to constant amplitude of $h_{k}$. The squeezing of the vacuum state leads to the emergence of classical properties of this state, as in the case of scalar metric fluctuations. 


\section{CONCEPTUAL PROBLEMS OF INFLATIONARY COSMOLOGY}

After this detailed survey of the theory of cosmological perturbations applied to inflationary cosmology we can now turn to some conceptual problems of cosmological inflation, and ways in which string theory may help address these issues. The first two problems relate to the cosmological perturbations we have just discussed.

The first problem concerns the amplitude of the cosmological fluctuations. Considering the simplest large-field potential

$$
V(\varphi)=\frac{1}{2} m^{2} \varphi^{2}
$$

the result (1.83) for the amplitude of the gravitational potential $\phi$ at late times and large scales (which modulo a factor of order unity gives the amplitude of the CMB fluctuations on large angular scales and hence should be of the order $10^{-5}$ ) yields (making use of the fact that the result from (1.83) must be evaluated for field values $\varphi \sim m_{p l}$ when the relevant scales exit the Hubble radius)

$$
\phi\left(t_{f}(k)\right) \sim \frac{m}{m_{p l}} .
$$

Hence, the value of $m$ must be chosen to be about $10^{13} \mathrm{GeV}$, introducing a new hierarchy problem into particle physics model building. In a model with quartic potential

$$
V(\varphi)=\frac{1}{4} \lambda \varphi^{4}
$$

we obtain a severe constraint on the value of the self coupling constant $\left(\lambda \ll 10^{-10}\right.$ modulo factors of $\left.2 \pi\right)$, a constraint which implies that the inflaton cannot be in thermal equilibrium before inflation. To reach this conclusion we have used "naturalness" considerations on coupling constants which state that the lower bound on the self coupling constant $\lambda$ implies lower bounds on the coupling constants describing interactions of $\varphi$ with other matter fields, since such interactions generate at higher loop order contributions to the renormalized value of $\lambda$. As shown in [83], this hierarchy problem is quite general.

As has recently been shown [59], this problem is worse in many inflationary models with entropy fluctuations. If the entropy perturbations are not suppressed during inflation, they can be parametrically amplified during reheating $[84,57,58,59]$. This results in fluctuations which are nonlinear after inflation independent of the values of the coupling constants, a result derived including the back-reaction of these fluctuations 
in the Hartree approximation [85]. Such models are thus phenomenologically ruled out.

The second problem is more important and will be discussed at length in the next section. Basically, since in most scalar field-driven inflationary models the period of inflation lasts much longer than the minimal number of e-foldings required in order that scales of current cosmological interest start out inside the Hubble radius at the beginning of inflation, in such models these scales thus originate with a wavelength much smaller than the Planck length, and hence the justification for using the formalism of the previous section to compute the evolution of fluctuations is doubtful. This is the trans-Planckian problem for inflationary cosmology [9] which becomes the trans-Planckian window of opportunity for string theory.

Scalar field-driven inflationary models have been shown to be geodesically incomplete in the past [86]. Hence, we know that this model cannot describe the very early Universe. A major challenge for string cosmology is to provide this description.

Most importantly, scalar field-driven inflation uses the time-independent part of $V(\varphi)$ to generate inflation. However, it is observationally known that (but theoretically not understood why) the time independent quantum vacuum contribution to the energy density of any quantum field does not gravitate. This is the famous cosmological constant problem. It may turn out that the solution of the cosmological constant problem will remove not only quantum vacuum energy but also the part of $V(\varphi)$ which generates inflation. I view this issue as the Achilles heel of scalar field-driven inflationary cosmology.

Finally, standard model particle physics does not provide a candidate for the inflaton. Models of particle physics beyond the standard model open the window for providing realizations of inflation.

Why can string theory help? First of all, string theory contains many fields which are massless in the early Universe, namely the moduli fields. Thus, it provides many candidates for an inflaton. In addition, it is quite possible that the hierarchy of scales required to give the right magnitude of density fluctuations emerges from a hierarchy of symmetry breaking scales in string theory. Secondly, string theory is supposed to describe the physics on all scales. Thus, in string cosmology the equations for evolving the fluctuations will be unambiguous (even if they are not known today), and the trans-Planckian problem will be solved. One of the main goals of string theory is to provide a nonsingular cosmology (and a concrete realization of this goal was provided in [87]). Thus, string theory should be able to provide a consistent theory of the very 
early Universe. This theory might connect with late-time cosmology through a period of inflation, or through scenarios more similar to $[7,8]$.

\section{THE TRANS-PLANCKIAN WINDOW FOR SUPERSTRING COSMOLOGY}

The same background dynamics which yields the causal generation mechanism for cosmological fluctuations, the most spectacular success of inflationary cosmology, bears in it the nucleus of the "trans-Planckian problem". This can be seen from Fig. (1.2). If inflation lasts only slightly longer than the minimal time it needs to last in order to solve the horizon problem and to provide a causal generation mechanism for CMB fluctuations, then the corresponding physical wavelength of these fluctuations is smaller than the Planck length at the beginning of the period of inflation. The theory of cosmological perturbations is based on classical general relativity coupled to a weakly coupled scalar field description of matter. Both the theories of gravity and of matter will break down on trans-Planckian scales, and this immediately leads to the trans-Planckian problem: are the predictions of standard inflationary cosmology robust against effects of trans-Planckian physics [9]?

This question has recently been addressed using a variety of techniques. The simplest method is to replace the usual dispersion relation for cosmological perturbations by an ad-hoc modified relation, as was done in $[88,89]$ in the context of studying the dependence of the thermal spectrum of black hole radiation on trans-Planckian physics. We will discuss the application of this method to cosmology [90, 91, 92] below. Other methods include considerations of modifications of the evolution of cosmological fluctuations coming from string-motivated space-space uncertainty relations [93, 94, 95, 96, 97], string-motivated space-time uncertainty relations [98] (reviewed below), from minimal length considerations [99], from an effective action analysis [100], from a minimal trans-Planckian physics viewpoint (starting each mode out in the vacuum state of the usual action for cosmological perturbations at the time when the physical wavelength is equal to the new fundamental length) $[101,102]$, and from the point of view of boundary renormalization group analysis [103].

The simplest way of modeling the possible effects of trans-Planckian physics [90, 91, 92] on the evolution of cosmological perturbations, while keeping the mathematical analysis simple, is to replace the linear dispersion relation $\omega_{\text {phys }}=k_{\text {phys }}$ of the usual equation for cosmological

perturbations by a non standard dispersion relation $\omega_{\text {phys }}=\omega_{\text {phys }}\left(k_{\text {phys }}\right)$ which differs from the standard one only for physical wavenumbers larger 


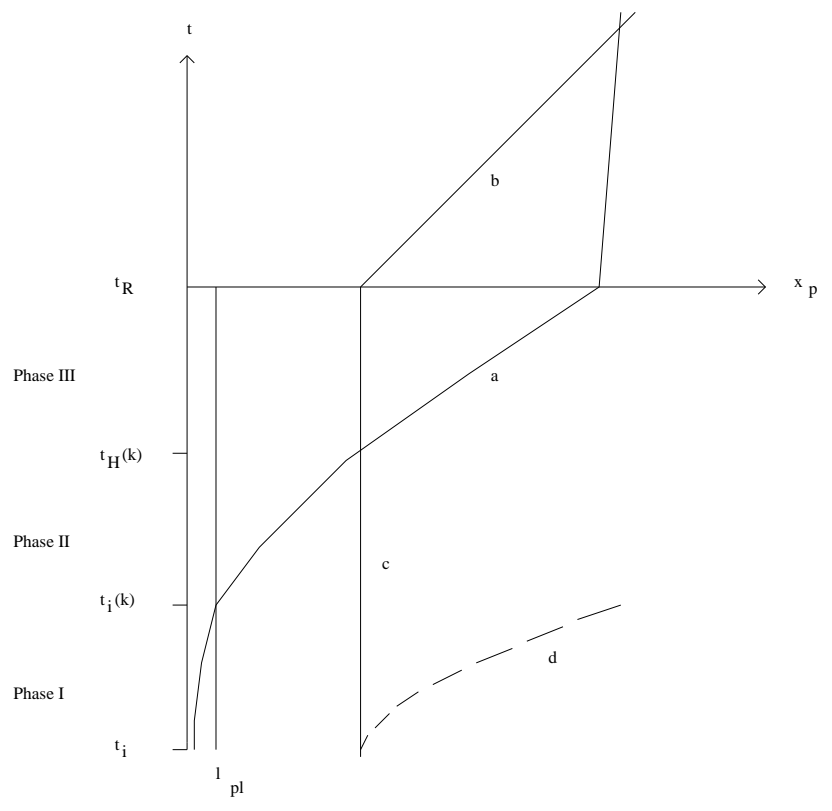

Figure 1.2 Space-time diagram (physical distance vs. time) showing the origin of the trans-Planckian problem of inflationary cosmology: at very early times, the wavelength is smaller than the Planck scale $\ell_{\mathrm{Pl}}$ (Phase I), at intermediate times it is larger than $\ell_{\mathrm{Pl}}$ but smaller than the Hubble radius $H^{-1}$ (Phase II), and at late times during inflation it is larger than the Hubble radius (Phase III). The line labeled a) is the physical wavelength associated with a fixed comoving scale $k$. The line b) is the Hubble radius or horizon in SBB cosmology. Curve c) shows the Hubble radius during inflation. The horizon in inflationary cosmology is shown in curve d). 
than the Planck scale. This amounts to replacing $k^{2}$ appearing in (1.93) with $k_{\text {eff }}^{2}(n, \eta)$ defined by

$$
k^{2} \rightarrow k_{\mathrm{eff}}^{2}(k, \eta) \equiv a^{2}(\eta) \omega_{\mathrm{phys}}^{2}\left(\frac{k}{a(\eta)}\right) .
$$

For a fixed comoving mode, this implies that the dispersion relation becomes time-dependent. Therefore, the equation of motion of the quantity $v_{k}(\eta)$ takes the form (with $z(\eta) \propto a(\eta)$ )

$$
v_{k}^{\prime \prime}+\left[k_{\text {eff }}^{2}(k, \eta)-\frac{a^{\prime \prime}}{a}\right] v_{k}=0 .
$$

A more rigorous derivation of this equation, based on a variational principle, has been provided [104] (see also Ref. [105]).

The evolution of modes thus must be considered separately in three phases, see Fig. (1.2). In Phase I the wavelength is smaller than the Planck scale, and trans-Planckian physics can play an important role. In Phase II, the wavelength is larger than the Planck scale but smaller than the Hubble radius. In this phase, trans-Planckian physics will have a negligible effect (this statement can be quantified [106]). Hence, by the analysis of the previous section, the wave function of fluctuations is oscillating in this phase,

$$
v_{k}=B_{1} \exp (-i k \eta)+B_{2} \exp (i k \eta)
$$

with constant coefficients $B_{1}$ and $B_{2}$. In the standard approach, the initial conditions are fixed in this region and the usual choice of the vacuum state leads to $B_{1}=1 / \sqrt{2 k}, B_{2}=0$. Phase III starts at the time $t_{\mathrm{H}}(k)$ when the mode crosses the Hubble radius. During this phase, the wave function is squeezed.

One source of trans-Planckian effects [90, 91] on observations is the possible non-adiabatic evolution of the wave function during Phase I. If this occurs, then it is possible that the wave function of the fluctuation mode is not in its vacuum state when it enters Phase II and, as a consequence, the coefficients $B_{1}$ and $B_{2}$ are no longer given by the standard expressions above. In this case, the wave function will not be in its vacuum state when it crosses the Hubble radius, and the final spectrum will be different. In particular, since short wavelength modes spend more time in the trans-Planckian phase, it is possible that a deviation of the spectrum from scale-invariance could be induced in a background which is expanding almost exponentially. This would be an order one effect on the spectrum of cosmological perturbations. In general, $B_{1}$ and $B_{2}$ are determined by the matching conditions between Phase I and II. If 
the dynamics is adiabatic throughout (in particular if the $a^{\prime \prime} / a$ term is negligible), the WKB approximation holds and the solution is always given by

$$
v_{k}(\eta) \simeq \frac{1}{\sqrt{2 k_{\text {eff }}(k, \eta)}} \exp \left(-i \int_{\eta_{\mathrm{i}}}^{\eta} k_{\text {eff }} \mathrm{d} \tau\right)
$$

where $\eta_{i}$ is some initial time. Therefore, if we start with a positive frequency solution only and use this solution, we find that no negative frequency solution appears. Deep in Region II where $k_{\text {eff }} \simeq k$ the solution becomes

$$
v_{k}(\eta) \simeq \frac{1}{\sqrt{2 k}} \exp (-i \phi-i k \eta)
$$

i.e. the standard vacuum solution times a phase which will disappear when we calculate the modulus. To obtain a modification of the inflationary spectrum, it is necessary to find a dispersion relation such that the WKB approximation breaks down in Phase I.

A concrete class of dispersion relations for which the WKB approximation breaks down is [89]

$$
k_{\text {eff }}^{2}(k, \eta)=k^{2}-k^{2}\left|b_{m}\right|\left[\frac{\ell_{\mathrm{pl}}}{\lambda(\eta)}\right]^{2 m},
$$

where $\lambda(\eta)=2 \pi a(\eta) / k$ is the wavelength of a mode. If we follow the evolution of the modes in Phases I, II and III, matching the mode functions and their derivatives at the junction times, the calculation [90, 91, 107] demonstrates that the final spectral index is modified and that superimposed oscillations appear. It has recently been shown [108] that in the case of this class of dispersion relations, the spectrum of black hole Hawking radiation is also affected.

However, the above example suffers from several problems. First, in inflationary models with a long period of inflationary expansion, the dispersion relation (1.116) leads to complex frequencies at the beginning of inflation for scales which are of current interest in cosmology. Furthermore, the initial conditions for the Fourier modes of the fluctuation field have to be set in a region where the evolution is non-adiabatic and the use of the usual vacuum prescription can be questioned. These problems can be avoided in a toy model in which we follow the evolution of fluctuations in a bouncing cosmological background which is asymptotically flat in the past and in the future. The analysis of [109] shows that even in this case the final spectrum of fluctuations depends on the specific dispersion relation used.

An example (see Fig. (1.3)) of a dispersion relation which breaks the WKB approximation in the trans-Planckian regime but does not lead to 


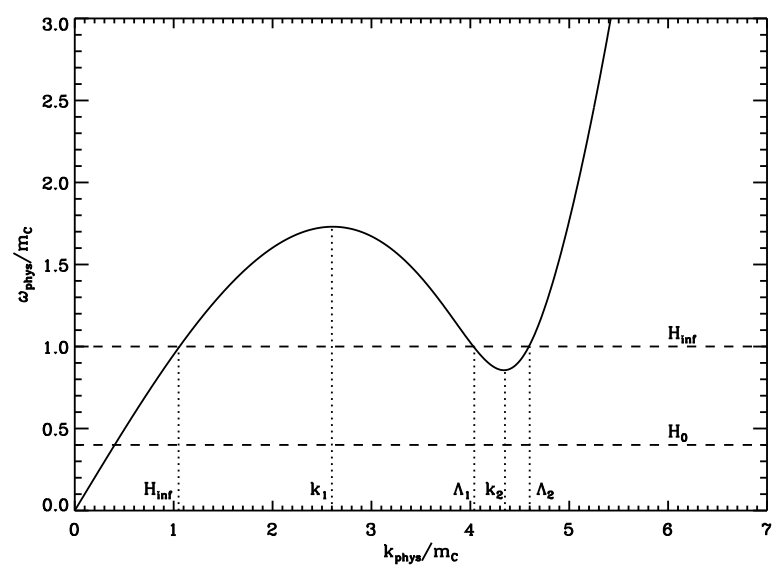

Figure 1.3 Sketch of the dispersion relation of [104]. The adiabaticity condition on the evolution of fluctuations is broken when the physical frequency (vertical axis) is smaller than the Hubble expansion rate. During the phase of inflation, this is the case when the physical wavenumber (horizontal axis) is in the interval between $\Lambda_{1}$ and $\Lambda_{2}$. Ultraviolet scales are $k>k_{0}$, and the value $k=k_{1}$ is the value when the dispersion relation turns over.

the problems mentioned in the previous paragraph was investigated in [104]. It is a dispersion relation which is linear for both small and large wavenumbers, but has an intermediate interval during which the frequency decreases as the wavenumber increases, much like what happens in (1.116). The violation of the WKB condition occurs for wavenumbers near the local minimum of the $\omega(k)$ curve. In this model, modes can be set up in the far ultraviolet in their Bunch-Davies vacuum. During the time interval when the physical wavenumber $k_{p}$ passes through the interval $\Lambda_{1}<k_{p}<\Lambda_{2}$ when the physical frequency is smaller than the value of the Hubble constant $H_{\text {inf }}$ in the inflationary phase, the mode is squeezed and thus is no longer in the local vacuum state when $k_{p}<\Lambda_{1}$.

A justified criticism against the method summarized in the previous analysis is that the non-standard dispersion relations used are completely ad hoc, without a clear basis in trans-Planckian physics. Other recently explored approaches are motivated by string theory. For example, there has been a lot of recent work [93, 94, 95, 96, 97] on the implication of space-space uncertainty relations $[110,111]$ for the evolution of fluctuations. The application of the uncertainty relations to the fluctuations lead to two effects: Firstly, the equation of motion of the fluctuations is modified. Secondly, for fixed comoving length scale $k$, the uncertainty relation is saturated at critical time $t_{i}(k)$. Thus, in addition to a modifi- 
cation of the evolution, trans-Planckian physics leads to a modification of the boundary conditions for the fluctuation modes. The upshot of this work is that the spectrum of fluctuations is modified. The magnitude of the deviations can be of the order $H / m_{p l}$, and is thus in principle measurable.

In [98], the implications of the stringy space-time uncertainty relation $[112,113]$

$$
\Delta x_{\text {phys }} \Delta t \geq l_{s}^{2}
$$

on the spectrum of cosmological fluctuations was studied. Again, application of this uncertainty relation to the fluctuations leads to two effects. Firstly, the coupling between the background and the fluctuations is nonlocal in time, thus leading to a modified dynamical equation of motion (a similar modification also results [114] from quantum deformations, another example of a consequence of non-commutative basic physics). Secondly, the uncertainty relation is saturated at the time $t_{i}(k)$ when the physical wavelength equals the string scale $l_{s}$. Before that time it does not make sense to talk about fluctuations on that scale. By continuity, it makes sense to assume that fluctuations on scale $k$ are created at time $t_{i}(k)$ in the local vacuum state (the instantaneous WKB vacuum state).

Let us for the moment neglect the nonlocal coupling between background and fluctuation, and thus consider the usual equation of motion for fluctuations in an accelerating background cosmology. We assume that $a(t)$ scales as a power of time, i.e. we consider power-law inflation, and we distinguish two ranges of scales. Ultraviolet modes are generated at late times when the Hubble radius is larger than $l_{s}$. On these scales, the spectrum of fluctuations does not differ from what is predicted by the standard theory, since at the time of Hubble radius crossing the fluctuation modes will be in their vacuum state. However, the evolution of the infrared modes which are created when the Hubble radius is smaller than $l_{s}$ is different. The fluctuations undergo less squeezing than they do in the absence of the uncertainty relation, and hence the final amplitude of fluctuations is lower. From the equation (1.98) for the power spectrum of fluctuations, and making use of the condition

$$
a\left(t_{i}(k)\right)=k l_{s}
$$

for the time $t_{i}(k)$ when the mode is generated, it follows immediately that the power spectrum is scale-invariant

$$
\mathcal{P}_{\mathcal{R}}(k) \sim k^{0} .
$$

In the standard scenario of power-law inflation the spectrum is red $\left(\mathcal{P}_{\mathcal{R}}(k) \sim k^{n-1}\right.$ with $\left.n<1\right)$. Taking into account the effects of the 
nonlocal coupling between background and fluctuation mode leads [98] to a modification of this result: the spectrum of fluctuations in a powerlaw inflationary background is in fact blue $(n>1)$.

Note that, if we neglect the nonlocal coupling between background and fluctuation mode, the result of (1.119) also holds in a cosmological background which is NOT accelerating. Thus, we have a method of obtaining a scale-invariant spectrum of fluctuations without inflation. This result was also subsequently obtained in [115], however without a micro-physical basis for the prescription for the initial conditions.

An approach to the trans-Planckian issue pioneered by Danielsson [101] which has recently received a lot of attention is to avoid the issue of the unknown trans-Planckian physics and to start the evolution of the fluctuation modes at the mode-dependent time when the wavelength equals the limiting scale. Obviously, the resulting spectrum will depend sensitively on which state is taken to be the initial state. The vacuum state is not unambiguous, and the choice of a state minimizing the energy density depends on the space-time splitting [102]. The signatures of this prescription are typically oscillations superimposed on the usual spectrum. The amplitude of this effect depends sensitively on the prescription of the initial state, and for a fixed prescription also on the background cosmology. For a discussion of these issues and a list of references on this approach the reader is referred to [116]. Note, in particular, that for a fixed background cosmology and for a fixed initial condition prescription, the amplitude of the correction terms in the spectrum may be different for scalar cosmological perturbations on one hand and gravitational waves or test scalar matter fields on the other hand.

If the ultraviolet modes are not in their vacuum state for wavelengths between the Planck or string scale and the Hubble radius, then the question of their back-reaction on the background geometry [117, 118, 119] arises. The naive expectation is that such ultraviolet modes have the equation of state of radiation, and that the mode occupation number hence must be very small (of order $\left(H_{\text {inf }} / m_{p l}\right)^{2}$ ) in order for the total energy density carried by these modes not to prevent inflation. This issue can be analyzed in the model of [104] in a setting in which there are no ultraviolet problems (in the naive approach one needs to assume that modes are continuously generated). In this approach, all modes start in their vacuum state, they are squeezed when $\Lambda_{1}<k_{p}<\Lambda_{2}$, and they oscillate at an excited level thereafter. In an exponentially expanding background it is clear by time-translation invariance that the energy density in the ultraviolet modes is constant. The stress-energy density of a test scalar field with this dispersion relation has recently been cal- 
culated [120], yielding the result that up to correction terms suppressed by $\left(H_{\text {inf }} / m_{p l}\right)^{2}$, the equation of state is that of a cosmological constant. Thus, the back-reaction of the excited states does not prevent inflation but rather leads to a renormalization of the cosmological constant. The back-reaction does effect the speed of rolling of the scalar field, and this yields constraints on the mode occupation numbers in the ultraviolet, constraints which, however, are much weaker than the ones conjectured in $[117,118,119]$.

Another constraint on trans-Planckian physics arises from the observational limits on the flux of ultra-high-energy cosmic rays. Such cosmic rays would be produced [121] in the present Universe if Trans-Planckian effects would lead to non-adiabatic mode evolution in the ultraviolet today. In the model of [104] this does not happen if the present Hubble rate $H_{0}$ is smaller than the local minimum of the dispersion relation, as in the situation depicted in Fig. (1.3).

In summary, due to the exponential red-shifting of wavelengths, present cosmological scales originate at wavelengths smaller than the Planck length early on during the period of inflation. Thus, Planck-scale physics may well encode information in these modes which can now be observed in the spectrum of microwave anisotropies. Two examples have been shown to demonstrate the existence of this "window of opportunity" to probe trans-Planckian physics in cosmological observations. The first method makes use of modified dispersion relations to probe the robustness of the predictions of inflationary cosmology, the second applies the stringy space-time uncertainty relation on the fluctuation modes. Both methods yield the result that trans-Planckian physics may lead to measurable effects in cosmological observables.

\section{CONCLUSIONS}

These lectures have focused on the quantum theory of the generation and evolution of cosmological fluctuations. This is the theory which connects the fundamental physics of the very early Universe with current cosmological observations. The general theory was illustrated in the context of the current paradigm of early universe cosmology, namely the inflationary universe scenario. In the context of the current approach to early universe cosmology which involves the coupling of matter described by quantum field theory to classical general relativity, inflationary cosmology suffers from important conceptual problems. Resolving these problems is a major goal for superstring cosmology. Thus, I would argue that inflationary cosmology requires string theory. 
Whether string theory requires inflation is a different issue. String theory provides many candidates for an inflaton, and thus it is possible (maybe even likely) that inflation can be implemented in string theory. The study of this issue has recently attracted a lot of attention. However, one should keep in mind that current observations do not prove the correctness of inflation. They show that the spectrum of fluctuations is nearly scale-invariant and nearly adiabatic. However, there are other ways to obtain such a spectrum, although the existing alternatives such as the ad hoc model mentioned in the previous section of these lecture notes, or the Pre-Big-Bang [7] and Ekpyrotic [8] scenarios are either not based on fundamental physics or not (yet) as developed as inflationary cosmology. Thus, it may be possible that string theory connects with observations using a cosmological scenario different than inflation. Even in this case, however, the theory of cosmological perturbations developed here is applicable.

A stringy early Universe may involve the dynamics of higher dimensions in an important way. This is the case for the Ekpyrotic scenario (see e.g. [122, 123] for some initial work). In this case, the formalism of cosmological perturbations needs to be extended (see e.g. [124] for a formalism which is a direct generalization of what was discussed here). There may be many interesting effects of bulk fluctuation modes which cannot be seen in a four-dimensional effective field theory approach. This is a rich field which merits much more work.

\section{Acknowledgments}

The author wishes to thank the organizers of this Cargèse school for the invitation to lecture and for their hospitality. He also wishes to thank Pei-Ming Ho and Jérôme Martin for collaborations on the issue of the trans-Planckian problem of inflationary cosmology. He is grateful to the Perimeter Institute for hospitality and financial support during the time when these lectures were prepared. He is supported in part by an NSERC Discovery grant (at McGill) and by the US Department of Energy under Contract DE-FG02-91ER40688, TASK A.

\section{References}

[1] C. L. Bennett et al., Astrophys. J. Suppl. 148, 1 (2003) [arXiv:astro$\mathrm{ph} / 0302207]$.

[2] M. Colless et al., arXiv:astro-ph/0306581.

[3] K. Abazajian et al. [SDSS Collaboration], Astron. J. 128, 502 (2004) [arXiv:astro-ph/0403325].

[4] A. H. Guth, Phys. Rev. D 23, 347 (1981). 
[5] A. Linde: Particle Physics and Inflationary Cosmology, (Harwood, Chur, 1990).

[6] V. Moncrief, J. Math. Phys. 17, 1893 (1976);

V. Moncrief, Prepared for Directions in General Relativity: An International Symposium in Honor of the 60th Birthdays of Dieter Brill and Charles Misner, College Park, MD, 27-29 May 1993

[7] M. Gasperini and G. Veneziano, M. Gasperini and G. Veneziano, Astropart. Phys. 1, 317 (1993) [arXiv:hep-th/9211021].

[8] J. Khoury, B. A. Ovrut, P. J. Steinhardt and N. Turok, Phys. Rev. D 64, 123522 (2001) [arXiv:hep-th/0103239].

[9] R. H. Brandenberger: Inflationary cosmology: Progress and problems. In: 1st Iranian International School on Cosmology: Large Scale Structure Formation, Kish Island, Iran, 22 Jan - 4 Feb 1999 Kluwer, Dordrecht, 2000. (Astrophys. Space Sci. Libr. ; 247), ed. by R. Mansouri and R. Brandenberger (Kluwer, Dordrecht, 2000). [arXiv:hep-ph/9910410].

[10] R. H. Brandenberger, Lect. Notes Phys. 646, 127 (2004) [arXiv:hepth/0306071].

[11] A. Vilenkin and E.P.S. Shellard; Cosmic Strings and Other Topological Defects, (Cambridge Univ. Press, Cambridge, 1994).

[12] M. B. Hindmarsh and T. W. Kibble, Rept. Prog. Phys. 58, 477 (1995) [arXiv:hep-ph/9411342].

[13] R. H. Brandenberger, Int. J. Mod. Phys. A 9, 2117 (1994) [arXiv:astro-ph/9310041].

[14] A. A. Starobinsky, Phys. Lett. B 91, 99 (1980).

[15] C. Armendariz-Picon, T. Damour and V. Mukhanov, Phys. Lett. B 458, 209 (1999) [arXiv:hep-th/9904075].

[16] R. H. Brandenberger and J. H. Kung, Phys. Rev. D 42, 1008 (1990).

[17] S. R. Coleman, Phys. Rev. D 15, 2929 (1977) [Erratum-ibid. D 16, 1248 (1977)];

C. G. . Callan and S. R. Coleman, Phys. Rev. D 16, 1762 (1977).

[18] R. H. Brandenberger, Rev. Mod. Phys. 57, 1 (1985).

[19] S. Dodelson, W. H. Kinney and E. W. Kolb, Phys. Rev. D 56, 3207 (1997) [arXiv:astro-ph/9702166].

[20] A. D. Linde, Phys. Lett. B 108, 389 (1982);

A. Albrecht and P. J. Steinhardt, Phys. Rev. Lett. 48, 1220 (1982).

[21] S. R. Coleman and E. Weinberg, Phys. Rev. D 7, 1888 (1973).

[22] D. S. Goldwirth and T. Piran, Phys. Rept. 214, 223 (1992). 
[23] R. Brandenberger, G. Geshnizjani and S. Watson, Phys. Rev. D 67, 123510 (2003) [arXiv:hep-th/0302222].

[24] A. D. Linde, Phys. Lett. B 129, 177 (1983).

[25] A. Linde, arXiv:hep-th/0402051.

[26] H. A. Feldman and R. H. Brandenberger, Phys. Lett. B 227, 359 (1989);

R. H. Brandenberger, H. Feldman and J. Kung, Phys. Scripta T36, 64 (1991).

[27] A. D. Linde, Phys. Rev. D 49, 748 (1994) [arXiv:astro-ph/9307002].

[28] J. Polchinski, arXiv:hep-th/0412244.

[29] A. D. Dolgov and A. D. Linde, Phys. Lett. B 116, 329 (1982).

[30] L. F. Abbott, E. Farhi and M. B. Wise, Phys. Lett. B 117, 29 (1982).

[31] J. H. Traschen and R. H. Brandenberger, Phys. Rev. D 42, 2491 (1990).

[32] L. Kofman, A. D. Linde and A. A. Starobinsky, Phys. Rev. Lett. 73, 3195 (1994) [arXiv:hep-th/9405187].

[33] Y. Shtanov, J. H. Traschen and R. H. Brandenberger, Phys. Rev. D 51, 5438 (1995) [arXiv:hep-ph/9407247].

[34] L. Kofman, A. D. Linde and A. A. Starobinsky, Phys. Rev. D 56, 3258 (1997) [arXiv:hep-ph/9704452].

[35] A. Liddle and D. Lyth, Cosmological Inflation and Large-Scale Structure, (Cambridge Univ. Press, Cambridge, 2000).

[36] V. F. Mukhanov, H. A. Feldman and R. H. Brandenberger, Phys. Rept. 215, 203 (1992).

[37] S. Weinberg: Gravitation and Cosmology, (Wiley, New York, 1972).

[38] P.J.E. Peebles: The Large-Scale Structure of the Universe, (Princeton Univ. Press, Princeton, 1980).

[39] T. Padmanabhan: Structure Formation in the Universe, (Cambridge Univ. Press, Cambridge, 1993).

[40] J. Peacock: Cosmological Physics, (Cambridge Univ. Press, Cambridge, 1999).

[41] R. K. Sachs and A. M. Wolfe, Astrophys. J. 147, 73 (1967).

[42] E. R. Harrison, Phys. Rev. D 1, 2726 (1970).

[43] Y. B. Zeldovich, Mon. Not. Roy. Astron. Soc. 160, 1 (1972).

[44] N. Afshordi and R. H. Brandenberger, Phys. Rev. D 63, 123505 (2001) [arXiv:gr-qc/0011075]. 
[45] E. Lifshitz, J. Phys. (USSR) 10, 116 (1946);

E. M. Lifshitz and I. M. Khalatnikov, Adv. Phys. 12, 185 (1963).

[46] J. M. Bardeen, Phys. Rev. D 22, 1882 (1980).

[47] W. Press and E. Vishniac, Astrophys. J. 239, 1 (1980).

[48] H. Kodama and M. Sasaki, Prog. Theor. Phys. Suppl. 78, 1 (1984).

[49] M. Bruni, G. F. Ellis and P. K. Dunsby, Class. Quant. Grav. 9, 921 (1992).

[50] J. c. Hwang, Astrophys. J. 415, 486 (1993).

[51] R. Durrer, Helv. Phys. Acta 69, 417 (1996).

[52] J. Stewart, Class. Quant. Grav. 7, 1169 (1990).

[53] J. Stewart and M. Walker, Proc. R. Soc. London A 341, 49 (1974).

[54] J. M. Bardeen, P. J. Steinhardt and M. S. Turner, Phys. Rev. D 28, 679 (1983).

[55] R. H. Brandenberger and R. Kahn, Phys. Rev. D 29, 2172 (1984).

[56] D. H. Lyth, Phys. Rev. D 31, 1792 (1985).

[57] F. Finelli and R. H. Brandenberger, Phys. Rev. Lett. 82, 1362 (1999) [arXiv:hep-ph/9809490].

[58] B. A. Bassett and F. Viniegra, Phys. Rev. D 62, 043507 (2000) [arXiv:hep-ph/9909353].

[59] F. Finelli and R. H. Brandenberger, Phys. Rev. D 62, 083502 (2000) [arXiv:hep-ph/0003172].

[60] J. H. Traschen, Phys. Rev. D 29, 1563 (1984).

[61] J. H. Traschen, N. Turok and R. H. Brandenberger, Phys. Rev. D 34, 919 (1986).

[62] G. Dvali, A. Gruzinov and M. Zaldarriaga, Phys. Rev. D 69, 023505 (2004) [arXiv:astro-ph/0303591].

[63] L. Kofman, arXiv:astro-ph/0303614.

[64] F. Vernizzi, Phys. Rev. D 69, 083526 (2004) [arXiv:astro$\mathrm{ph} / 0311167]$.

[65] M. Axenides, R. H. Brandenberger and M. S. Turner, Phys. Lett. B 126, 178 (1983).

[66] S. Weinberg, Phys. Rev. D 67, 123504 (2003) [arXiv:astro$\mathrm{ph} / 0302326]$.

[67] W. B. Lin, X. H. Meng and X. M. Zhang, Phys. Rev. D 61, 121301 (2000) [arXiv:hep-ph/9912510].

[68] V. F. Mukhanov and G. V. Chibisov, JETP Lett. 33, 532 (1981) [Pisma Zh. Eksp. Teor. Fiz. 33, 549 (1981)]. 
[69] A. H. Guth and S. Y. Pi, Phys. Rev. Lett. 49, 1110 (1982).

[70] A. A. Starobinsky, Phys. Lett. B 117, 175 (1982).

[71] S. W. Hawking, Phys. Lett. B 115, 295 (1982).

[72] V. N. Lukash, Pisma Zh. Eksp. Teor. Fiz. 31, 631 (1980);

V. N. Lukash, Sov. Phys. JETP 52, 807 (1980) [Zh. Eksp. Teor. Fiz. 79, (1980)].

[73] W. Press, Phys. Scr. 21, 702 (1980).

[74] K. Sato, Mon. Not. Roy. Astron. Soc. 195, 467 (1981).

[75] V. F. Mukhanov, JETP Lett. 41, 493 (1985) [Pisma Zh. Eksp. Teor. Fiz. 41, 402 (1985)].

[76] V. F. Mukhanov, Sov. Phys. JETP 67, 1297 (1988) [Zh. Eksp. Teor. Fiz. 94N7, 1 (1988 ZETFA,94,1-11.1988)].

[77] M. Sasaki, Prog. Theor. Phys. 76, 1036 (1986).

[78] N. Birrell and P.C.W. Davies: Quantum Fields in Curved Space, (Cambridge Univ. Press, Cambridge, 1982).

[79] R. H. Brandenberger, Nucl. Phys. B 245, 328 (1984).

[80] R. H. Brandenberger and C. T. Hill, Phys. Lett. B 179, 30 (1986).

[81] D. Polarski and A. A. Starobinsky, Class. Quant. Grav. 13, 377 (1996) [arXiv:gr-qc/9504030].

[82] L. P. Grishchuk, Sov. Phys. JETP 40, 409 (1975) [Zh. Eksp. Teor. Fiz. 67, 825 (1974)].

[83] F. C. Adams, K. Freese and A. H. Guth, Phys. Rev. D 43, 965 (1991).

[84] B. A. Bassett, D. I. Kaiser and R. Maartens, Phys. Lett. B 455, 84 (1999) [arXiv:hep-ph/9808404].

[85] J. P. Zibin, R. H. Brandenberger and D. Scott, Phys. Rev. D 63, 043511 (2001) [arXiv:hep-ph/0007219].

[86] A. Borde and A. Vilenkin, Phys. Rev. Lett. 72, 3305 (1994) [arXiv:gr-qc/9312022].

[87] R. H. Brandenberger and C. Vafa, Nucl. Phys. B 316, 391 (1989).

[88] W. G. Unruh, Phys. Rev. D51, 2827 (1995).

[89] S. Corley and T. Jacobson, Phys. Rev. D54, 1568 (1996) [arXiv:hepth/9601073].

[90] J. Martin and R. H. Brandenberger, Phys. Rev. D63, 123501 (2001) [arXiv:hep-th/0005209].

[91] R. H. Brandenberger and J. Martin, Mod. Phys. Lett. A16, 999 (2001) [arXiv:astro-ph/0005432]. 
[92] J. C. Niemeyer, Phys. Rev. D63, 123502 (2001) [arXiv:astro$\mathrm{ph} / 0005533]$.

[93] C. S. Chu, B. R. Greene and G. Shiu, Mod. Phys. Lett. A 16, 2231 (2001) [arXiv:hep-th/0011241].

[94] R. Easther, B. R. Greene, W. H. Kinney and G. Shiu, Phys. Rev. D64, 103502 (2001) [arXiv:hep-th/0104102].

[95] R. Easther, B. R. Greene, W. H. Kinney and G. Shiu, Phys. Rev. D 67, 063508 (2003) [arXiv:hep-th/0110226].

[96] F. Lizzi, G. Mangano, G. Miele and M. Peloso, JHEP 0206, 049 (2002) [arXiv:hep-th/0203099].

[97] S. F. Hassan and M. S. Sloth, Nucl. Phys. B 674, 434 (2003) [arXiv:hep-th/0204110].

[98] R. Brandenberger and P. M. Ho, Phys. Rev. D66, 023517 (2002) [arXiv:hep-th/0203119].

[99] A. Kempf and J. C. Niemeyer, Phys. Rev. D64, 103501 (2001) [arXiv:astro-ph/0103225].

[100] C. P. Burgess, J. M. Cline, F. Lemieux and R. Holman, JHEP 0302, 048 (2003) [arXiv:hep-th/0210233];

C. P. Burgess, J. M. Cline and R. Holman, JCAP 0310, 004 (2003) [arXiv:hep-th/0306079].

[101] U. H. Danielsson, Phys. Rev. D 66, 023511 (2002) [arXiv:hepth/0203198];

U. H. Danielsson, JHEP 0207, 040 (2002) [arXiv:hep-th/0205227].

[102] V. Bozza, M. Giovannini and G. Veneziano, JCAP 0305, 001 (2003) [arXiv:hep-th/0302184].

[103] K. Schalm, G. Shiu and J. P. van der Schaar, JHEP 0404, 076 (2004) [arXiv:hep-th/0401164].

[104] M. Lemoine, M. Lubo, J. Martin and J. P. Uzan, Phys. Rev. D65, 023510 (2002) [arXiv:hep-th/0109128].

[105] T. Jacobson and D. Mattingly, Phys. Rev. D63, 041502 (2001) [arXiv:hep-th/0009052].

[106] N. Kaloper, M. Kleban, A. E. Lawrence and S. Shenker, Phys. Rev. D 66, 123510 (2002) [arXiv:hep-th/0201158].

[107] J. Martin and R. H. Brandenberger, Phys. Rev. D65, 103514 (2002) [arXiv:hep-th/0201189].

[108] W. G. Unruh and R. Schutzhold, arXiv:gr-qc/0408009.

[109] R. H. Brandenberger, S. E. Joras and J. Martin, Phys. Rev. D 66, 083514 (2002) [arXiv:hep-th/0112122]. 
[110] D. Amati, M. Ciafaloni and G. Veneziano, Phys. Lett. B197, 81 (1987).

[111] D. J. Gross and P. F. Mende, Nucl. Phys. B303, 407 (1988).

[112] T. Yoneya, Mod. Phys. Lett. A4, 1587 (1989).

[113] M. Li and T. Yoneya, arXiv:hep-th/9806240.

[114] S. Cremonini, Phys. Rev. D 68, 063514 (2003) [arXiv:hepth/0305244].

[115] S. Hollands and R. M. Wald, Gen. Rel. Grav. 34, 2043 (2002) [arXiv:gr-qc/0205058].

[116] J. Martin and R. Brandenberger, Phys. Rev. D 68, 063513 (2003) [arXiv:hep-th/0305161].

[117] T. Tanaka, arXiv:astro-ph/0012431.

[118] A. A. Starobinsky, Pisma Zh. Eksp. Teor. Fiz. 73, 415 (2001) [JETP Lett. 73, 371 (2001)] [arXiv:astro-ph/0104043].

[119] M. Porrati, Phys. Lett. B 596, 306 (2004) [arXiv:hep-th/0402038].

[120] R. H. Brandenberger and J. Martin, arXiv:hep-th/0410223.

[121] A. A. Starobinsky and I. I. Tkachev, JETP Lett. 76, 235 (2002) [Pisma Zh. Eksp. Teor. Fiz. 76, 291 (2002)] [arXiv:astro$\mathrm{ph} / 0207572]$.

[122] A. J. Tolley, N. Turok and P. J. Steinhardt, Phys. Rev. D 69, 106005 (2004) [arXiv:hep-th/0306109].

[123] T. J. Battefeld, S. P. Patil and R. Brandenberger, Phys. Rev. D 70, 066006 (2004) [arXiv:hep-th/0401010].

[124] C. van de Bruck, M. Dorca, R. H. Brandenberger and A. Lukas, Phys. Rev. D 62, 123515 (2000) [arXiv:hep-th/0005032]. 